\title{
Calibrating the Cepheid period-Iuminosity relation from the infrared surface brightness technique
}

\section{The $\boldsymbol{p}$-factor, the Milky Way relations, and a universal $\boldsymbol{K}$-band relation ${ }^{\star}$}

\author{
J. Storm ${ }^{1}$, W. Gieren ${ }^{2}$, P. Fouqué ${ }^{3}$, T. G. Barnes ${ }^{4}$, G. Pietrzyński²,5, \\ N. Nardetto ${ }^{2,6}$, M. Weber ${ }^{1}$, T. Granzer $^{1}$, and K. G. Strassmeier ${ }^{1}$ \\ ${ }^{1}$ Leibniz-Institut für Astrophysik Potsdam (AIP), An der Sternwarte 16, 14482 Potsdam, Germany \\ e-mail: jstorm@aip.de \\ ${ }^{2}$ Universidad de Concepción, Departamento de Astronomía, Casilla 160-C, Concepción, Chile \\ 3 IRAP, Université de Toulouse, CNRS, 14 Av. E. Belin, 31400 Toulouse, France \\ ${ }^{4}$ University of Texas at Austin, McDonald Observatory, 82 Mt. Locke Rd, McDonald Observatory, TX 79734, USA \\ 5 Warsaw University Observatory, Al. Ujazdowskie 4, 00-478 Warsaw, Poland \\ ${ }^{6}$ Laboratoire Fizeau, UNS/OCA/CNRS UMR6525, Parc Valrose, 06108 Nice Cedex 2, France
}

Received 28 April 2011 / Accepted 23 July 2011

\section{ABSTRACT}

\begin{abstract}
Aims. We determine period-luminosity relations for Milky Way Cepheids in the optical and near-IR bands. These relations can be used directly as reference for extra-galactic distance determination to Cepheid populations with solar metallicity, and they form the basis for a direct comparison with relations obtained in exactly the same manner for stars in the Magellanic Clouds, presented in an accompanying paper. In that paper we show that the metallicity effect is very small and consistent with a null effect, particularly in the near-IR bands, and we combine here all 111 Cepheids from the Milky Way, the LMC and SMC to form a best relation.

Methods. We employ the near-IR surface brightness (IRSB) method to determine direct distances to the individual Cepheids after we have recalibrated the projection factor using the recent parallax measurements to ten Galactic Cepheids and the constraint that Cepheid distances to the LMC should be independent of pulsation period.

Results. We confirm our earlier finding that the projection factor for converting radial velocity to pulsational velocity depends quite steeply on pulsation period, $p=1.550-0.186 \log (P)$ in disagrement with recent theoretical predictions. We find PL relations based on 70 Milky Way fundamental mode Cepheids of $M_{K}=-3.33( \pm 0.09)(\log (P)-1.0)-5.66( \pm 0.03), W_{V I}=-3.26( \pm 0.11)(\log (P)-1.0)-$ 5.96( \pm 0.04$)$. Combining the 70 Cepheids presented here with the results for 41 Magellanic Cloud Cepheids which are presented in an accompanying paper, we find $M_{K}=-3.30( \pm 0.06)(\log (P)-1.0)-5.65( \pm 0.02), W_{V I}=-3.32( \pm 0.08)(\log (P)-1.0)-5.92( \pm 0.03)$. Conclusions. We delineate the Cepheid PL relation using 111 Cepheids with direct distances from the IRSB analysis. The relations are by construction in agreement with the recent HST parallax distances to Cepheids and slopes are in excellent agreement with the slopes of apparent magnitudes versus period observed in the LMC.
\end{abstract}

Key words. stars: variables: Cepheids - stars: fundamental parameters - stars: distances - distance scale

\section{Introduction}

In this series of papers we calibrate the Cepheid periodluminosity (PL-) relation using the infrared surface brightness (IRSB) method. In Paper II we address the effect of metallicity on both the slope and the zero point of the relations in both the optical and near-IR bands finding very small (consistent with zero) effects in the near-IR and small, but possibly significant effects in the optical.

Gieren et al. (2005) made a first determination of the LMC PL relations based on thirteen stars with IRSB based distances. They found that the distances to the individual Cepheids were dependent on the pulsation period which of course is unphysical. They found that the problem could be resolved by changing the adopted projection $(p-)$ factor relation, which converts observed

* Full Table 3 is only available at the CDS via anonymous ftp to cdsarc.u-strasbg.fr $(130.79 .128 .5)$ or via

http://cdsarc.u-strasbg.fr/viz-bin/qcat?J/A+A/534/A94 radial velocities into pulsation velocities that are needed for Baade-Wesselink type analysis.

In the present paper we use the new and largely expanded data set from Paper II for now 36 LMC Cepheids together with the new direct geometric parallax measurements from Benedict et al. (2007) to empirically determine the appropriate $p$-factor relation to be used in the analyses.

We present new accurate radial velocity data for 14 galactic Cepheids expanding the sample to a total of 77 Cepheids, 70 of which can be used to delineate the Milky Way PL relations. We have reanalyzed the complete sample using exactly the same code and calibrations as for the LMC sample and adopting exactly the same reddening law to allow a direct comparison.

Based on the (near-) universality of the PL relations we combine the Milky Way, LMC and SMC samples to determine PL relations based on 111 Cepheids which at the same time constrain the slopes very well, and which are tied directly to the parallax zero point from Benedict et al. (2007). These relations thus form a very solid basis for the Cepheid distance scale. 
The paper is structured as follows: in Sect. 2 we present the data from the literature as well as new radial velocity data for fourteen Milky Way Cepheids. In Sect. 3 we present the IRSB method and use the Benedict et al. (2002, 2007) parallaxes as well as the results from Paper II on 36 LMC Cepheids with IRSB distances to constrain the $p$-factor relation. We then use the new $p$-factor relation to determine distances and luminosities for 77 Cepheids and derive new PL relations for the 70 fundamental mode pulsators with good data sets. We proceed to combine the data with the Magellanic Cloud sample to give our best global PL relations which can be used for distance determination to other galaxies. In Sect. 4 we compare the results with other recent investigations and in Sect. 5 we summarize our conclusions.

\section{The data}

We have searched the literature for optical ( $V$-band) and nearinfrared ( $K$-band) light curves as well as radial velocity curves. The starting point for the search was the catalogues of complete phase coverage $K$-band light curves for Milky Way Cepheids published by Welch et al. (1984), Laney \& Stobie (1992) and Barnes et al. (1997). Since the publication of those papers a rich literature of high quality optical and radial velocity data has materialized and for the majority of the stars it is now possible to apply the near-infrared surface brightness method to determine distances and luminosities.

In addition to the literature data we have obtained new, accurate, radial velocity curves for 14 of these Cepheids to improve the phase coverage and/or data quality for these stars.

We have selected the data sets according to quality and completeness, but also to ensure, as far as possible, that the data have been obtained close in time to the near-infrared data to reduce possible errors due to period variations of the stars. Known double mode pulsators have been disregarded a priori as the application of the IRSB method to such stars could only be attempted if all the data were truly simultaneous. Thus the list of references reflects this pre-selection and does not include the data sets that were not used for the analysis. A number of first overtone pulsators has also been included, but they are of course not used for the delineation of the fundamental mode pulsator PL relations.

In Table 1 we present the list of stars and the references to the data sets which we have used in the present analysis. A more complete list of of data sets can be found in Groenewegen (2008).

The $B V I_{\mathrm{c}}$ photometry reported here is all on the JohnsonKron-Cousins system and the different data sets have been transformed to this system when necessary. Similarly all the near-IR data have been transformed to the SAAO system as necessary using the transformations from Carter (1990).

The radial velocity data have all been obtained using high resolution $(R>20000)$ spectrographs. Most of the radial velocities have been derived using cross-correlation techniques or techniques which are equivalent. In this way the radial velocities are assumed to be on a common system and we have not seen indications of significant systematic differences between datasets for any of our stars for which we have had data from different techniques. This is an important point for the application of the IRSB method as the conversion from radial velocities to pulsational velocities, the so called $p$-factor, depends to some extent on the procedure which was used for deriving the radial velocity from the input data.

\subsection{New radial velocity measurements}

For fourteen of the stars the radial velocity curves were either missing or they were of limited quality. We have obtained 381 new radial velocity measurements for these stars (see Table 2) using the STELLA Echelle Spectrograph (SES) mounted on the fully robotic $1.2 \mathrm{~m}$ STELLA-I telescope (Strassmeier et al. 2004, 2010; and Weber et al. 2008) at the Izaña Observatory on Tenerife, Spain. SES is a fiber-fed echelle spectrograph with a $2 \mathrm{k} \times 2 \mathrm{k}$ CCD detector covering the wavelength range from 388 to $882 \mathrm{~nm}$ with small inter-order gaps starting at $732 \mathrm{~nm}$ and increasing towards the red. The resolving power is $R=55000$ giving a spectral resolution of $0.12 \AA$ at $650 \mathrm{~nm}$.

The spectra were obtained in fully robotic mode in the period from March 2007 until July 2010 and reduced using the automatic data reduction pipeline (Ritter \& Wasshüttl 2004; Weber et al. 2008) developed for the instrument.

The radial velocities returned by the pipeline were corrected for instrumental velocity offsets and placed on the CORAVEL system by applying the offset of $+0.503 \mathrm{~km} \mathrm{~s}^{-1}$ determined by Strassmeier et al. (in prep.) The radial velocities are tabulated in Table 3 and plotted in Fig. 1.

\subsection{Pulsation velocities}

The $p$-factor (see Sect. 3.4), which is used to convert the observed radial velocities into pulsational velocities, depends to some extent on the spectrograph and the procedure used for extracting the velocities as different spectral features might carry different weight in deriving the pulsational velocity. To investigate this effect for the STELLA velocities we have observed the star TT Aql for which an excellent CORAVEL based velocity curve is available (Imbert 1999).

We follow the procedure described by Storm et al. (2004) to determine the ratio between the $p$-factors for STELLA and CORAVEL based velocities, $\eta_{\text {STELLA }}=p_{\text {STELLA }} / p_{\text {COR }}$, for the relevant phase interval, $0 \leq \phi \leq 0.8$. In Fig. 2 we plot the difference in pulsational velocity,

$$
\Delta V_{\mathrm{puls}}=p_{\mathrm{COR}}\left(\mathrm{RV}_{\mathrm{COR}}-V_{\gamma}\right)-\eta p_{\mathrm{COR}}\left(\mathrm{RV}_{\mathrm{STELLA}}-V_{\gamma}\right)
$$

as a function of phase between the linearly interpolated observed radial velocities for the two spectrographs for three different values of $\eta$. It appears that in the phase interval from 0.15 to 0.75 where the velocity difference curve is smooth, the formally best value is $\eta=1.03$. It is however also clear that the uncertainty is rather large and given that the datasets have been obtained at epochs differing by about ten years, we choose not to apply any additional corrections to the STELLA velocities but assume that the STELLA and CORAVEL $p$-factors agree to within 3\%. For the present sample of 14 stars with STELLA velocities a $3 \%$ effect on the $p$-factor translates into a $1 \%$ effect on the slopes of the final PL relations which is much smaller than the statistical errors.

For some stars we have used the radial velocity data from Nardetto et al. (2009) using the HARPS data pipeline. This data set also contains data for a star, $\zeta \mathrm{Gem}$, for which a good CORAVEL data set is available from Bersier (1994). Unfortunately the period of the star is not perfectly constant over time so it has been necessary to shift the two radial velocity curves with respect to each other to obtain a good match. Proceeding then as for the STELLA dataset we find that the two data sets are in good agreement and that $\eta_{\text {HARPS }}=1.00 \pm 0.03$ as can be seen in Fig. 3 . 
Table 1. List of data sources.

\begin{tabular}{|c|c|c|c|c|c|c|c|}
\hline ID & Optical & $K$-band & Rad. vel. & ID & Optical & $K$-band & Rad. vel. \\
\hline$\eta \mathrm{Aql}$ & 4,17 & 3,4 & $9,12,29,35$ & U Nor & $7,16,20$ & 2 & 8,21 \\
\hline U Aql & 17 & 3 & 32 & QZ Nor & 6,47 & 2 & 28,41 \\
\hline FF Aql & 13,17 & 3 & 53 & TW Nor & 16,20 & $2,3,58$ & 23,41 \\
\hline FM Aql & 4,17 & 3,4 & 35 & V340 Nor & 42 & 2 & 23 \\
\hline FN Aql & 17,20 & 3 & 34 & Y Oph & $7,17,20$ & 2 & 27,55 \\
\hline SZ Aql & 17,20 & 2 & 35,40 & BF Oph & 10,17 & 2 & $11,14,15$ \\
\hline TT Aql & $4,7,13,17,20$ & 3,4 & 1 & X Pup & 17,20 & 2 & 1 \\
\hline V496 Aql & $10,17,47$ & 3 & 1,48 & AQ Pup & 7,17 & $2,22,24$ & 1 \\
\hline RT Aur & 13,17 & 4 & 12,34 & BN Pup & $7,16,20$ & 2,22 & 1 \\
\hline$\ell$ Car & 20,38 & 2 & 40 & LS Pup & 7 & 2,22 & 1 \\
\hline U Car & 7,20 & 2,3 & 8,40 & RS Pup & $17,20,47$ & 2 & 9 \\
\hline V Car & 20 & 2 & 15 & VZ Pup & 7,20 & 2,22 & 1 \\
\hline CT Car & 51 & 2,22 & $21,41,47$ & KQ Sco & 7 & 2,3 & 8,54 \\
\hline VY Car & 7 & 2 & 8,15 & RY Sco & $7,16,17,20$ & 2 & $8,14,15$ \\
\hline WZ Car & 7,20 & 2,22 & 8 & EV Sct & 17,20 & 2 & $9,19,23$ \\
\hline SU Cas & 4,17 & 4 & 9,23 & RU Sct & 17,20 & 2,3 & 19,41 \\
\hline KN Cen & 7 & 2 & 8,21 & SS Sct & 17,20 & 3 & 54 \\
\hline V Cen & 10,20 & 2 & 11,15 & S Sge & $4,13,17$ & 3,4 & 34 \\
\hline VW Cen & 7,20 & 2 & 8 & GY Sge & 43 & 2 & 19,34 \\
\hline XX Cen & 39 & 2 & 8 & U Sgr & 10,17 & 2 & 9,23 \\
\hline$\delta$ Cep & 4,17 & 4 & $9,12,23,29,35$ & W Sgr & 17,20 & $3,59,60$ & 23 \\
\hline SU Cru & 20 & 2 & 8,21 & X Sgr & 17,20 & 3,57 & 1 \\
\hline X Cyg & 13,17 & 3,4 & 9,29 & Y Sgr & 17,20 & 3 & 1,56 \\
\hline CD Cyg & 17 & 3 & 26 & BB Sgr & 10,17 & 2 & 1 \\
\hline DT Cyg & 17 & 3 & 23 & WZ Sgr & 17 & 2,3 & 34 \\
\hline SU Cyg & 13,17 & 3 & 31 & XX Sgr & 17 & 3 & 1 \\
\hline VZ Cyg & 4,17 & 3,4 & 1 & YZ Sgr & 17,47 & 3 & 1,48 \\
\hline$\beta$ Dor & 20,33 & 2 & 37 & V350 Sgr & 10,17 & 3 & $11,27,34$ \\
\hline$\zeta$ Gem & 13 & 57 & 1 & SZ Tau & 4,17 & 2,4 & 23 \\
\hline X Lac & 4,17 & 4 & 23 & $\mathrm{~T}$ Vel & 47,20 & 2 & 11,15 \\
\hline Y Lac & 4,17 & 4 & 26 & CS Vel & 44,45 & 2,22 & 23,41 \\
\hline Z Lac & 4,17 & 4 & 36 & RY Vel & 7,16 & 2,3 & 8,21 \\
\hline BG Lac & 4,17 & 4 & 26 & RZ Vel & $7,16,20$ & 2 & 8,56 \\
\hline GH Lup & 7,20 & 2 & 8 & SW Vel & $7,16,20$ & 2 & 21,27 \\
\hline T Mon & 47,7 & 2 & 9,23 & S Vul & 43 & 2,3 & 34 \\
\hline CV Mon & 17,20 & 2 & 9,41 & T Vul & 13,17 & 3,4 & 23 \\
\hline S Mus & 46 & 2,3 & 30 & U Vul & $4,13,17$ & 4 & 23,25 \\
\hline UU Mus & 16,39 & 2 & 8 & SV Vul & 13,17 & 2,3 & 23,26 \\
\hline S Nor & 44,46 & 2 & 23 & & & & \\
\hline
\end{tabular}

References. (1) This paper; (2) Laney \& Stobie (1992); (3) Welch, et al. (1984); (4) Barnes et al. (1997); (6) Berdnikov \& Turner (1995); (7) Coulson, \& Caldwell (1985); (8) Coulson et al. (1985); (9) Storm et al. (2004); (10) Gieren (1981b); (11) Gieren (1981a); (12) Kiss (1998b); (13) Kiss (1998a); (14) Barnes et al. (1988); (15) Lloyd Evans (1980); (16) Madore (1975); (17) Moffett \& Barnes (1984); (19) Metzger et al. (1991); (20) Pel (1976); (21) Pont et al. (1994); (22) Schechter et al. (1992); (23) Bersier et al. (1994); (24) Welch (1985); (25) Imbert (1996); (26) Imbert (1999); (27) Petterson et al. (2005); (28) Kienzle et al. (1999); (29) Butler \& Bell (1997); (30) Evans (1990); (31) Imbert (1984); (32) Welch et al. (1987); (33) Shobbrook (1992); (34) Gorynya et al. (1998); (35) Barnes et al. (2005); (36) Sugars \& Evans (1996); (37) Wallerstein et al. (1992); (38) Bersier (2002); (39) Coulson et al. (1985); (40) Bersier (2002); (41) Metzger et al. (1992); (42) Bersier et al. (1994); (43) Berdnikov (1986, 1987, 1992, 1992, 1992, 1992, 1992); (44) Berdnikov \& Turner (1998); (45) Berdnikov \& Turner (2000); (46) Walraven et al. (1964); (47) Berdnikov \& Caldwell (2001); (48) Caldwell et al. (2001); (51) Pojmanski et al.; (53) Evans (1990); (54) Groenewegen (2008); (55) Nardetto et al. (2006); (56) Nardetto et al. (2009); (57) Feast et al. (2008); (58) McGonegal et al. (1983); (59) Kimeswenger et al. (2004); (60) Wisniewski \& Johnson (1968).

Table 2. Cepheids for which we have obtained new radial velocity curves.

\begin{tabular}{ll|rl}
\hline \hline Star & $N_{\text {obs }}$ & Star & $N_{\text {obs }}$ \\
\hline TT Aql & 25 & LS Pup & 26 \\
V496 Aql & 19 & VZ Pup & 29 \\
C Gem & 65 & X Sgr & 19 \\
VZ Cyg & 20 & Y Sgr & 15 \\
X Pup & 42 & BB Sgr & 18 \\
AQ Pup & 38 & XX Sgr & 20 \\
BN Pup & 31 & YZ Sgr & 14 \\
\hline
\end{tabular}

Notes. For each star the number of new data points is listed as well.
For another star, $\ell$ Car, there is a similar possibility of a direct comparison between CORAVEL and HARPS data. The radial velocity curves from different data sets exhibit some variations though, and the conclusions are less straight forward than for $\zeta$ Gem, but they do agree with a value of $\eta$ which is indistinguishable from unity, which we will adopt in the following.

\section{The analysis}

\subsection{The IRSB method}

The infrared surface-brightness (IRSB) method is a variant of the Baade-Wesselink method originally developed by 

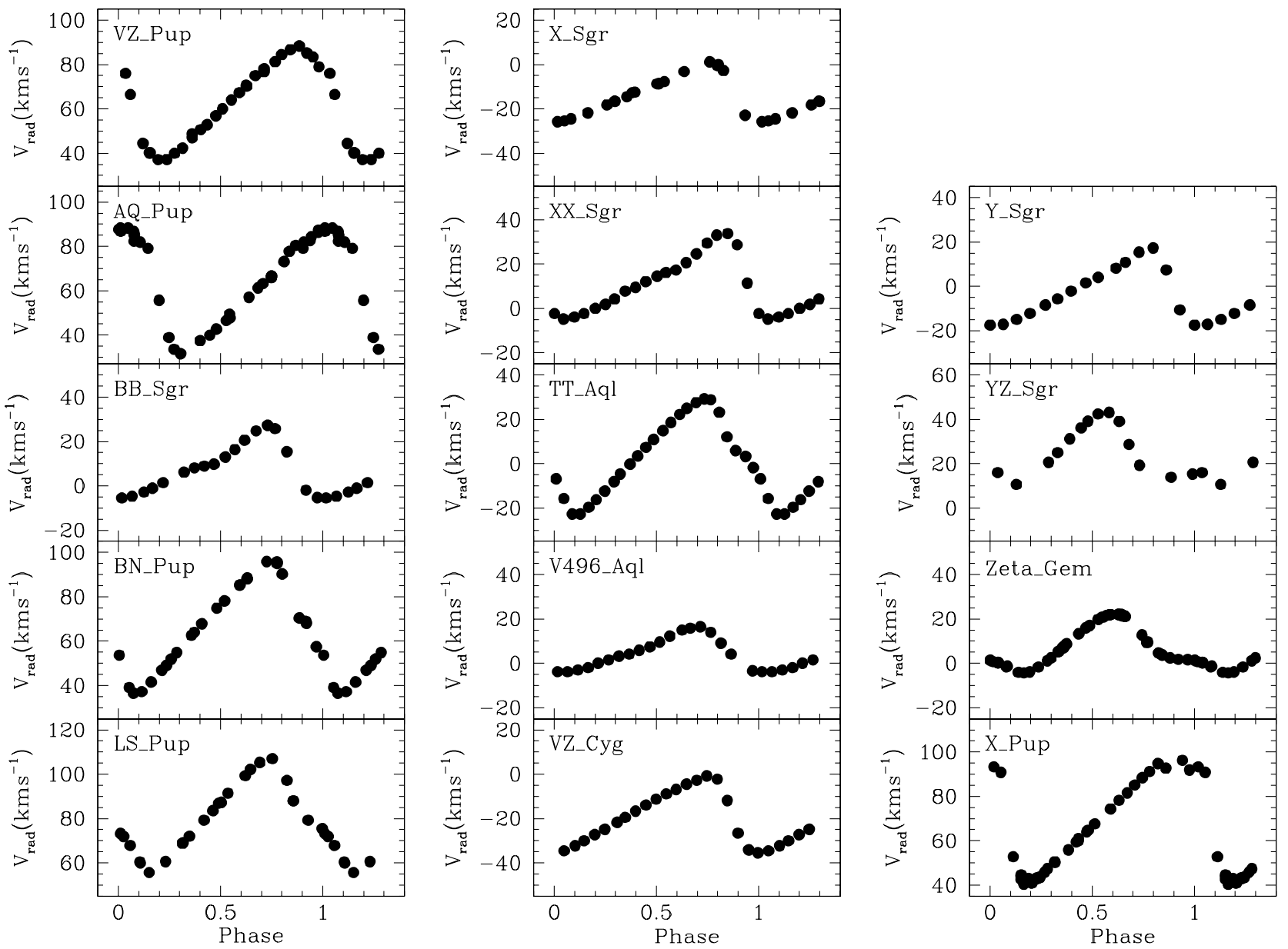

Fig. 1. The new radial velocity curves for fourteen Milky Way Cepheids from the STELLA echelle spectrograph as tabulated in Table 3.

Table 3. Heliocentric radial velocities (RV) measured with the STELLA echelle Spectrograph (SES) as returned by the data reduction pipeline and offset to the CORAVEL velocity zero point.

\begin{tabular}{lcrrr}
\hline \hline Star & $\begin{array}{c}\text { HJD } \\
(\text { Days })\end{array}$ & Phase & $\begin{array}{c}\text { RV } \\
\left(\mathrm{km} \mathrm{s}^{-1}\right)\end{array}$ & $\begin{array}{r}\sigma(\mathrm{RV}) \\
\left(\mathrm{km} \mathrm{s}^{-1}\right)\end{array}$ \\
\hline TTAq1 & 2454175.76609 & 0.53 & 14.79 & 0.04 \\
TTAq1 & 2454213.73669 & 0.29 & -8.15 & 0.06 \\
TTAq1 & 2454218.64326 & 0.65 & 24.95 & 0.07 \\
TTAq1 & 2454222.59739 & 0.94 & 3.25 & 0.02 \\
TTAq1 & 2454223.59473 & 0.01 & -6.92 & 0.02 \\
$\ldots$ & $\ldots$ & $\ldots$ & $\ldots$ & $\ldots$ \\
\hline
\end{tabular}

Notes. The complete table is available in electronic from the CDS.

Barnes \& Evans (1976) in the optical wavelengths. It is based on a functional relation between a colour index and the surface brightness parameter in the $V$-band, $F_{V}$. It was originally calibrated by Welch (1994) and a few years later Fouqué \& Gieren (1997). They determined a very tight linear relationship between the $(V-K)$ colour index and $F_{V}$ based on interferometric angular diameters of giant stars found in the literature, thus extending the method to the near-infrared. The scatter in this relation was significantly smaller than was the case for the optical colour indices used previously. A detailed description of the implementation of the method which we use in the present paper can be found in Storm et al. (2004).

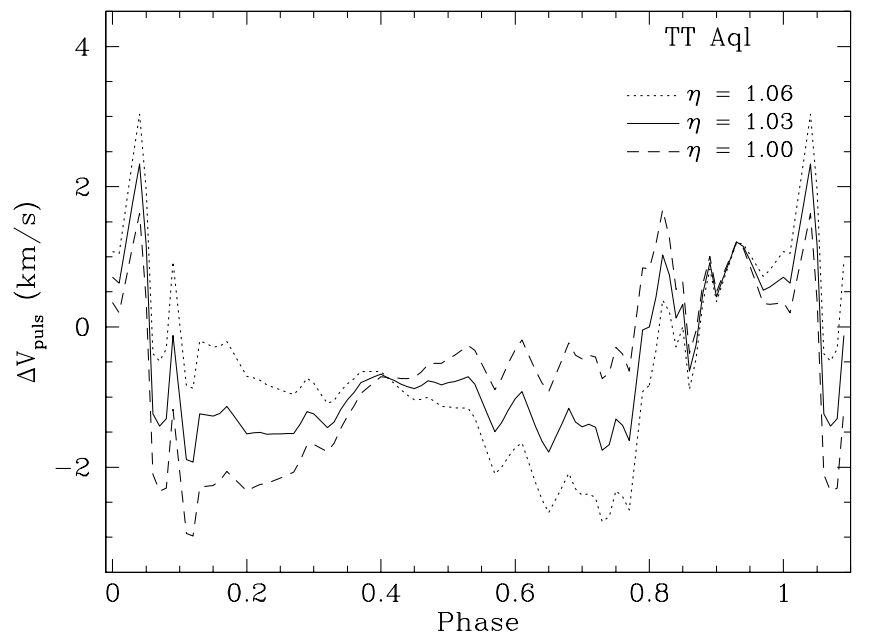

Fig. 2. The difference in pulsational velocity as a function of phase between CORAVEL and STELLA measurements for three different choices of $\eta$ where $\eta=p_{\text {STELLA }} / p_{\text {COR }}$.

Recently direct interferometric angular diameter measurements of Cepheids have become available (Nordgren et al. 2002; Kervella et al. 2004a; Mérand et al. 2005) allowing a direct comparison between the surface-brightness relation for static stars with actual pulsating stars. On this basis Kervella et al. (2004b) find excellent agreement between static and pulsating 
J. Storm et al.: Calibrating the Cepheid period-luminosity relation from the infrared surface brightness technique. I.

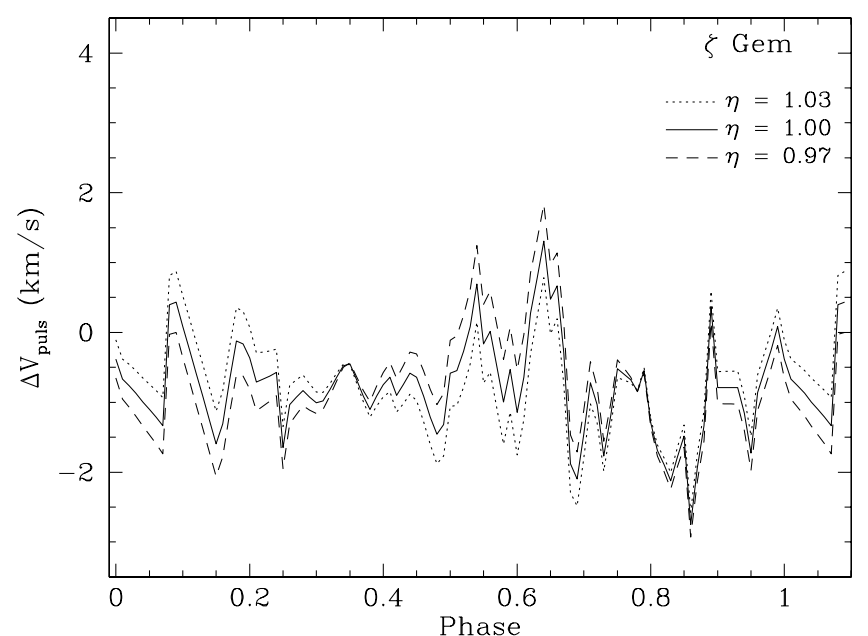

Fig. 3. The difference in pulsational velocity as a function of phase between CORAVEL and HARPS measurements for three different choices of $\eta$ where $\eta=p_{\mathrm{HARPS}} / p_{\mathrm{COR}}$.

stars as well as with the relation determined by Fouqué \& Gieren (1997) for static stars. They find a best fit relation of

$F_{V}=-0.1336(V-K)_{0}+3.9530$

with the coefficients determined to better than $2 \%$. We adopt their relation for the following analysis.

The surface brightness measure $F_{V}$ is directly related to the stellar angular diameter, $\theta$ through the relation

$F_{V}(\phi)=4.2207-0.1 V_{0}(\phi)-0.5 \log \theta(\phi)$

where $V_{0}$ is the de-reddened visual magnitude, and $\phi$ is the phase.

At the same time geometry gives us the stellar angular diameter from the stellar radius, $R(\phi)$, through the relation

$\theta(\phi)=2 R(\phi) / d=2\left(R_{0}+\Delta R(\phi)\right) / d$

where $\phi$ is the pulsation phase, $d$ is the distance and $R$ is the radius.

Integrating the radial velocity curve then gives the radius variation, $\Delta R(\phi)$ between a reference radius, $R_{0}$ and the given phase, $\phi$ as

$\Delta R(\phi)=\int-p\left[V_{r}(\phi)-V_{\gamma}\right] \mathrm{d} \phi$

where $p$ is the so called projection factor converting radial velocity into pulsational velocity, $V_{r}(\phi)$ is the observed radial velocity and $V_{\gamma}$ is the systemic velocity.

We can now solve Eq. (4) for the two parameters, mean radius, $R_{0}$, and distance, $d$ by linear regression to the observed values of $\theta(\phi)$ from Eq. (3) and $\Delta R(\phi)$ from Eq. (5). As discussed in Storm et al. (2004) we use the OLS bi-sector fit from Isobe et al. (1990) for the regression fit. We fit only the phase interval $\phi \in[0.0,0.8]$ where the shapes of the two curves usually agrees very well, and we avoid the phase region $\phi \in[0.8,1.0]$ where the agreement often is quite poor, most likely due to shocks in the stellar atmosphere. We also allow for a small phase shift between the photometric and radial velocity data to optimize the quality
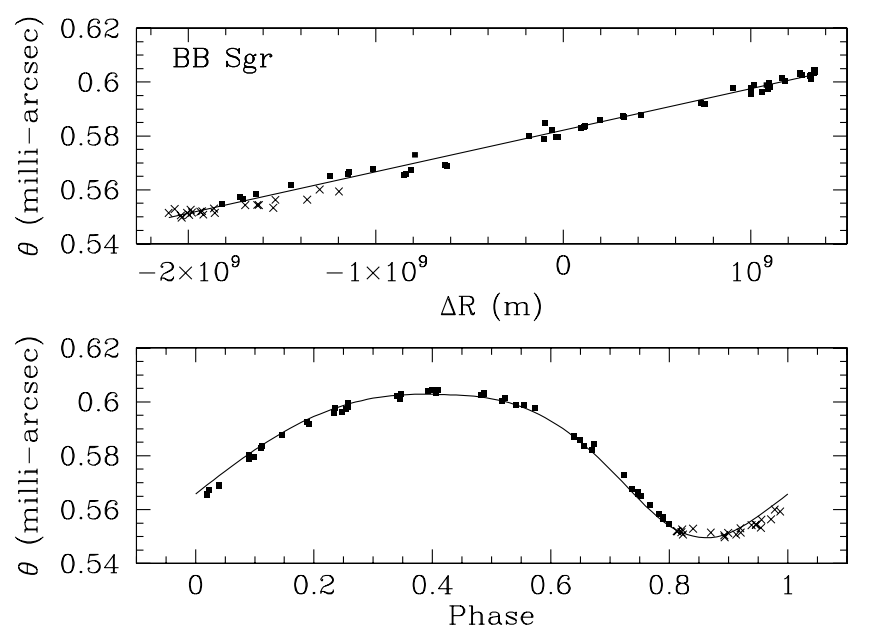

Fig. 4. The IRSB fit to the data for the star BB Sgr. The deviation between photometric and spectroscopic angular diameters in the phase interval $\phi \in[0.8,1.0]$ is evident and is observed for many of the Cepheids in our sample. This phase interval is therefore disregarded in the fit for all the stars.

of the fit. The effect on the final PL relation of these phase shifts is mainly to decrease the scatter in the relation.

An example of the fit for the star BB Sgr is shown in Fig. 4. In the upper panel the data used for the actual OLS bi-sector fit (see Storm et al. 2004, for more details on this) can be seen and in the lower panel the corresponding photometric angular diameters have been plotted as filled squares for the points used in the fit and red crosses for the points in the phase interval $\phi \in[0.8,1.0]$ which have been disregarded in the fits. The curve in the lower panel delineates the corresponding spectroscopic angular diameter.

\subsection{Absorption}

In order to derive dereddened magnitudes for our Cepheids we use the colour excess values as given in Fouqué et al. (2007). These values are on the system defined by Laney \& Caldwell (2007) and as discussed by Fouqué et al. (2007), these values are in agreement with the system established by Tammann et al. (2003), who recalibrated the original measurements compiled by Fernie (1995).

For the reddening law we similarly adopt the choice made by Fouqué et al. (2007), namely the law determined by Cardelli et al. (1989) with a total-to-selective absorption in the $V$ band of $R_{V}=3.23$ as determined by Sandage et al. (2004). For the other bands we use $A_{I}=0.608 \times A_{V}, A_{K}=0.119 \times A_{V}, A_{J}=0.292 \times A_{V}$, and $A_{H}=0.181 \times A_{V}$.

\subsection{Fourier coefficients and identification of fundamental mode pulsators}

Before we attempt to establish the PL relations we have to identify the fundamental mode pulsators in the sample. This is difficult without referring to the period-luminosity diagram. We use the Fourier parameters for the radial velocity data and the diagrams from Kienzle et al. (1999) to reject overtone pulsators. To be conservative we use the $A_{1}$ parameter (the radial velocity amplitude) to reject $s$-type Cepheids from our final sample as well. In this way we might remove some bona fide fundamental pulsators as well, but we ensure that we have a uniform sample. 


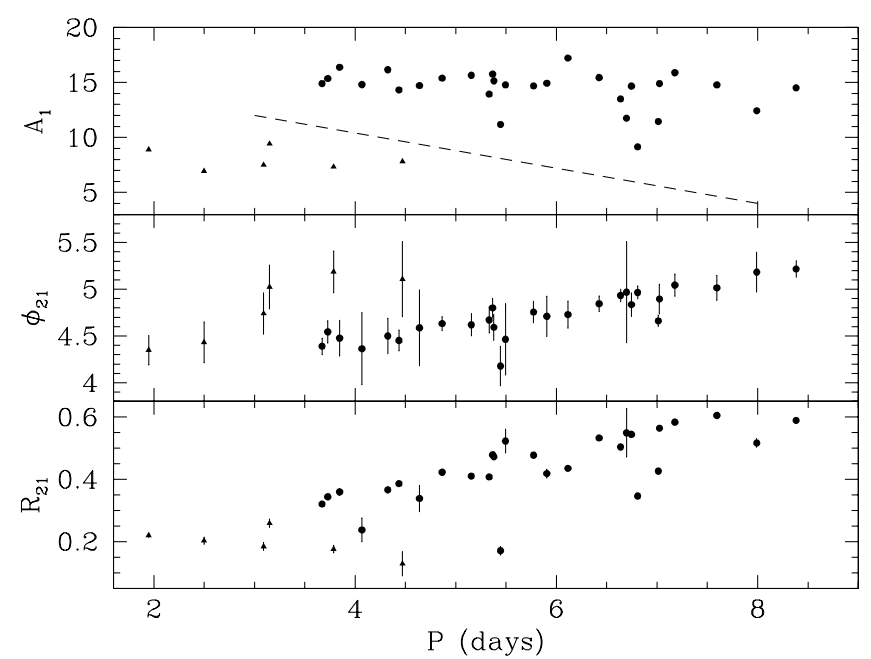

Fig. 5. The Fourier parameters based on the radial velocity data for the Cepheids with periods less than 9 days. The dashed line indicates the adopted division between normal Cepheids and $s$-Cepheids based on Fig. 2 in Kienzle et al. (1999). Filled circles indicate fundamental pulsators and the triangles, first overtone pulsators.

The Fourier parameters for all the Cepheids based on the data sets given in Table 1 are tabulated in Table 4 . The error estimates on the parameters have been calculated using the approximative formula given by Petersen (1986).

In Fig. 5 the Fourier parameters for the short period Cepheids have been plotted and the $s$-Cepheids identified as the stars with $A_{1}$ below the dashed line. These stars are DT Cyg, EV Sct, FF Aql, SU Cas, SZ Tau, and QZ Nor. SU Cas is the shortestperiod Cepheid in our sample and was probably the first Galactic Cepheid for which pulsation in the first overtone mode was firmly established by Gieren $(1976,1982)$. Two stars lie below the sequence of fundamental mode pulsators in the $A_{1}$ vs. $P$ plot but above the dashed line and in the other Fourier parameters they appear unremarkable, so they are not obvious $s$-Cepheids or overtone pulsators. These stars are X Lac, and V496 Aql and we will keep them in the sample of fundamental mode stars.

\subsection{Constraining the projection factor}

For any Baade-Wesselink type method it is necessary to determine the pulsational velocity of the surface of the star as this is the velocity curve which is matched against the angular diameter curve from the photometry. The same is the case when the angular diameter curve is directly determined from interferometry. The conversion from the observed radial velocity to pulsational velocity is commonly parametrized with the projection factor, $p$.

The $p$-factor is largely a geometrical correction taking into account the fact that the radial velocity that we measure is based on light coming from all points of the hemisphere of the star which is visible to the observer and not just from the surface element which move along the line of sight to the star. In fact as discussed by Sabbey et al. (1995) the $p$-factor depends on the temperature of the star, which changes with pulsation period, as it depends on the limb darkening of the star.

The $p$-factor used for converting the observed radial velocities into pulsational velocities has direct consequences for the derived distances, as it scales directly with the stellar radius variation, and is probably the largest source of systematic error for the method. In the past we (e.g. Gieren et al. 1993; Storm et al. 2004; Barnes et al. 2005) have used a relation with a weak period dependence, $p=1.39-0.03 \log P$, based on theoretical work by Hindsley \& Bell (1986).

Later we (Gieren et al. 2005) found that the use of this $p$ factor relation with the IRSB method for LMC Cepheids leads to an unphysical dependence of the distance modulus on the pulsation period. We found that a $p$-factor relation of $p=$ $1.58-0.15 \log P$ removed this period effect, but the conclusion was not very firm due to the limited sample of only 13 LMC Cepheids.

On the theoretical side, Nardetto et al. (2007) have carefully analyzed individual line profiles from pulsating atmosphere models and they also found a relation which was steeper than the Hindsley \& Bell (1986) slope. Nardetto et al. (2009) generalized this work to match the cross-correlation technique used in most observational work on radial velocities and found a relation

$$
p=1.31( \pm 0.06)-0.08( \pm 0.05) \log P .
$$

With the sample of 36 LMC Cepheids, presented in Storm et al. (2011, hereinafter Paper II), covering a wide range of periods we are in a much better position to constrain the $p$-factor relation empirically. Furthermore the recent direct parallax measurements for ten Milky Way Cepheids by Benedict et al. (2002, 2007) using the Hubble Space Telescope Fine Guidance Sensor provide a fundamental set of reference data which we can use to calibrate the $p$-factor relation.

For three of the ten stars with HST parallaxes (Y Sgr, X Sgr, and $\zeta$ Gem) we present new radial velocities here, significantly improving the available data quality. For one star, W Sgr, which is a known binary (Szabados 2003) the IRSB fit is clearly very poor and we disregard this star in the further analysis. X Sgr is known to be affected by a double shockwave in its atmosphere (Mathias et al. 2006) but the IRSB fit looks fine and the agreement with the HST parallax is excellent so we keep it in the sample. This leaves us with nine stars in common.

As a first step we use the theoretical relation from Nardetto et al. (2009) in Eq. (6) and apply the IRSB method to these nine stars. We find a disappointing difference of $-0.30 \pm 0.05$ mag in the distance moduli, the IRSB distances being shorter. Applying the Nardetto et al. relation to the LMC Cepheids in Paper II we similarly find an unlikely result, namely an LMC distance modulus of $18.26 \pm 0.04$, much shorter than the canonical value of 18.50. So if we proceed using first principles, we have a serious conflict with the Benedict et al. (2007) result as well as with most recent works on the LMC distance that have confined the true distance modulus to a value between 18.4 and 18.6 (e.g. Pietrzyński et al. 2009; Szewczyk et al. 2008).

To reconcile these results we have to conclude either that the theoretical $p$-factor relation is incorrect due to the lack of some physics, or that there is a period dependence in the IRSB method which is not properly accounted for in the current theoretical $p$ factor relation. We attempt to constrain this effect empirically and parametrize it as a part of the $p$-factor relation which we retain as having the simple linear form $p=\alpha_{p} \times \log (P)+\beta_{p}$.

We need to determine two parameters, namely the slope $\left(\alpha_{p}\right)$ and the zero point $\left(\beta_{p}\right)$ of the $p$-factor relation. We have two independent constraints, namely that there should be no systematic dependence of the LMC Cepheid distances with pulsation period, and we should reproduce, on average, the Benedict et al. (2007) distances.

It turns out that these two constraints are largely orthogonal in the $\left(\beta_{p}, \alpha_{p}\right)$ plane as can be seen in Fig. 6 . To determine these parameters we simply apply the IRSB method to each sample of stars (Milky Way and LMC) for an array of slopes 
J. Storm et al.: Calibrating the Cepheid period-luminosity relation from the infrared surface brightness technique. I.

Table 4. Fourier parameters for the stars based on the radial velocity data.

\begin{tabular}{|c|c|c|c|c|c|c|c|c|c|c|c|}
\hline ID & $\begin{array}{l}\text { Period } \\
\text { (day) }\end{array}$ & $\begin{array}{c}\text { Systemic velocity } \\
\left(\mathrm{km} \mathrm{s}^{-1}\right)\end{array}$ & $\begin{array}{r}A_{1} \\
\left(\mathrm{~km} \mathrm{~s}^{-1}\right)\end{array}$ & $R_{21}$ & $R_{31}$ & $\phi_{21}$ & $\phi_{31}$ & $\bar{\sigma} \sigma\left(R_{21}\right)$ & $\bar{\sigma} \sigma\left(R_{31}\right)$ & $\sigma\left(\phi_{21}\right)$ & $\sigma\left(\phi_{31}\right)$ \\
\hline SU Cas & 1.94932 & -7.36 & 8.90 & 0.220 & 0.053 & 4.348 & 2.576 & 0.006 & 0.006 & 0.157 & 0.235 \\
\hline DT Cyg & 2.49921 & -1.70 & 6.93 & 0.203 & 0.045 & 4.434 & 2.067 & 0.012 & 0.012 & 0.218 & 0.326 \\
\hline EV Sct & 3.09099 & 17.26 & 7.50 & 0.185 & 0.046 & 4.741 & 2.748 & 0.012 & 0.012 & 0.221 & 0.330 \\
\hline SZ Tau & 3.14895 & -0.65 & 9.42 & 0.259 & 0.075 & 5.024 & 3.784 & 0.013 & 0.013 & 0.233 & 0.346 \\
\hline SS Sct & 3.67125 & -7.24 & 14.90 & 0.298 & 0.097 & 4.449 & 2.616 & 0.002 & 0.002 & 0.096 & 0.143 \\
\hline RT Aur & 3.72832 & 19.57 & 15.36 & 0.319 & 0.145 & 4.618 & 3.057 & 0.004 & 0.004 & 0.131 & 0.194 \\
\hline QZ Nor & 3.78655 & -39.50 & 7.33 & 0.176 & 0.043 & 5.186 & 4.732 & 0.012 & 0.012 & 0.223 & 1.855 \\
\hline SU Cyg & 3.84549 & 0.08 & 16.38 & 0.334 & 0.147 & 4.545 & 2.836 & 0.010 & 0.010 & 0.209 & 0.303 \\
\hline BF Oph & 4.06751 & -28.90 & 14.81 & 0.222 & 0.137 & 4.420 & 3.110 & 0.035 & 0.035 & 0.423 & 0.580 \\
\hline Y Lac & 4.32378 & -22.20 & 16.16 & 0.340 & 0.192 & 4.570 & 2.884 & 0.010 & 0.010 & 0.208 & 0.303 \\
\hline T Vul & 4.43541 & -2.90 & 14.32 & 0.358 & 0.162 & 4.517 & 2.829 & 0.004 & 0.004 & 0.123 & 0.185 \\
\hline FF Aql & 4.47085 & -0.09 & 7.83 & 0.129 & 0.057 & 5.108 & 3.615 & 0.039 & 0.039 & 0.400 & 0.593 \\
\hline $\mathrm{T} \mathrm{Vel}$ & 4.63982 & 5.75 & 14.72 & 0.314 & 0.103 & 4.665 & 3.037 & 0.038 & 0.038 & 0.446 & 0.615 \\
\hline VZ Cyg & 4.86445 & -18.30 & 15.39 & 0.392 & 0.155 & 4.716 & 3.302 & 0.002 & 0.002 & 0.082 & 0.123 \\
\hline V350 Sgr & 5.15424 & 7.73 & 15.65 & 0.380 & 0.193 & 4.701 & 3.320 & 0.004 & 0.004 & 0.129 & 0.191 \\
\hline BG Lac & 5.33191 & -18.10 & 13.94 & 0.378 & 0.194 & 4.758 & 3.431 & 0.006 & 0.006 & 0.155 & 0.230 \\
\hline$\delta$ Cep & 5.36630 & -16.40 & 15.76 & 0.443 & 0.293 & 4.900 & 3.381 & 0.003 & 0.003 & 0.110 & 0.163 \\
\hline CV Mon & 5.37879 & 19.28 & 15.15 & 0.437 & 0.212 & 4.672 & 3.219 & 0.005 & 0.005 & 0.152 & 0.225 \\
\hline X Lac & 5.44453 & -25.20 & 11.18 & 0.161 & 0.022 & 4.216 & 2.001 & 0.013 & 0.013 & 0.233 & 0.344 \\
\hline V Cen & 5.49392 & -23.60 & 14.78 & 0.483 & 0.190 & 4.531 & 2.691 & 0.035 & 0.035 & 0.418 & 0.583 \\
\hline Y Sgr & 5.77335 & -1.57 & 14.68 & 0.442 & 0.237 & 4.851 & 3.593 & 0.004 & 0.004 & 0.129 & 0.192 \\
\hline CS Vel & 5.90474 & 26.85 & 14.93 & 0.388 & 0.171 & 4.802 & 3.522 & 0.013 & 0.013 & 0.237 & 0.346 \\
\hline FM Aql & 6.11423 & -5.01 & 17.22 & 0.403 & 0.198 & 4.822 & 3.821 & 0.006 & 0.006 & 0.159 & 0.234 \\
\hline XX Sgr & 6.42414 & 12.65 & 15.44 & 0.492 & 0.205 & 4.949 & 3.904 & 0.002 & 0.002 & 0.092 & 0.136 \\
\hline BB Sgr & 6.63714 & 8.40 & 13.50 & 0.466 & 0.152 & 5.047 & 4.346 & 0.001 & 0.001 & 0.076 & 0.113 \\
\hline V Car & 6.69668 & 12.60 & 11.75 & 0.508 & 0.165 & 5.085 & 4.615 & 0.071 & 0.071 & 0.595 & 0.819 \\
\hline U Sgr & 6.74523 & 2.70 & 14.67 & 0.503 & 0.190 & 4.939 & 4.005 & 0.005 & 0.005 & 0.139 & 0.206 \\
\hline V496 Aql & 6.80703 & 5.10 & 9.15 & 0.322 & 0.063 & 5.082 & 4.625 & 0.001 & 0.001 & 0.076 & 0.114 \\
\hline X Sgr & 7.01283 & -12.70 & 11.45 & 0.395 & 0.148 & 4.748 & 3.459 & 0.001 & 0.001 & 0.065 & 0.098 \\
\hline U Aql & 7.02410 & -0.11 & 14.90 & 0.521 & 0.183 & 5.005 & 4.279 & 0.007 & 0.007 & 0.176 & 0.261 \\
\hline$\eta \mathrm{Aql}$ & 7.17678 & -14.80 & 15.89 & 0.539 & 0.246 & 5.169 & 4.062 & 0.004 & 0.004 & 0.131 & 0.194 \\
\hline W Sgr & 7.59503 & -27.70 & 14.78 & 0.559 & 0.189 & 5.136 & 3.921 & 0.005 & 0.005 & 0.146 & 0.217 \\
\hline U Vul & 7.99069 & 0.24 & 12.43 & 0.478 & 0.104 & 5.321 & 3.970 & 0.013 & 0.013 & 0.236 & 0.349 \\
\hline S Sge & 8.38209 & -13.40 & 14.51 & 0.544 & 0.115 & 5.358 & 4.086 & 0.002 & 0.002 & 0.098 & 0.146 \\
\hline GH Lup & 9.27787 & -16.10 & 4.38 & 0.146 & 0.000 & 5.823 & 0.000 & 0.183 & 0.183 & 0.870 & -1.634 \\
\hline FN Aql & 9.48151 & 12.67 & 14.60 & 0.163 & 0.156 & 0.566 & 5.571 & 0.005 & 0.005 & 0.137 & 0.232 \\
\hline YZ Sgr & 9.55369 & 22.88 & 13.74 & 0.444 & 0.122 & 0.016 & 4.347 & 0.002 & 0.002 & 0.083 & 0.127 \\
\hline S Mus & 9.66007 & -0.14 & 12.85 & 0.479 & 0.137 & 5.820 & -2.260 & 0.011 & 0.011 & 0.209 & 0.338 \\
\hline S Nor & 9.75424 & 5.54 & 14.31 & 0.332 & 0.161 & 0.255 & -1.444 & 0.007 & 0.007 & 0.168 & 0.373 \\
\hline$\beta$ Dor & 9.84308 & 8.87 & 14.01 & 0.313 & 0.192 & 0.337 & 4.914 & 0.017 & 0.017 & 0.263 & 0.626 \\
\hline$\zeta$ Gem & 10.15073 & 7.44 & 11.81 & 0.243 & 0.137 & 0.459 & 5.046 & 0.001 & 0.001 & 0.066 & 0.099 \\
\hline TW Nor & 10.78618 & -56.60 & 18.78 & 0.193 & 0.218 & 0.685 & 5.181 & 0.013 & 0.013 & 0.233 & 1.316 \\
\hline Z Lac & 10.88564 & 0.09 & 18.43 & 0.180 & 0.172 & 0.209 & 5.059 & 0.008 & 0.008 & 0.181 & 0.269 \\
\hline XX Cen & 10.95337 & -16.20 & 14.23 & 0.340 & 0.140 & 0.308 & 5.238 & 0.051 & 0.051 & 0.474 & 0.689 \\
\hline V340 Nor & 11.28700 & -40.30 & 8.58 & 0.265 & 0.053 & 0.206 & 4.441 & 0.016 & 0.016 & 0.413 & 0.391 \\
\hline UU Mus & 11.63641 & -16.90 & 20.12 & 0.200 & 0.205 & 0.049 & 5.724 & 0.044 & 0.044 & 0.462 & 69.984 \\
\hline U Nor & 12.64371 & -22.20 & 16.88 & 0.135 & 0.087 & 0.206 & 4.921 & 0.044 & 0.044 & 0.448 & 0.646 \\
\hline SU Cru & 12.84760 & -30.30 & 11.06 & 0.009 & 0.096 & 3.232 & 5.500 & 0.067 & 0.067 & 1.272 & 0.792 \\
\hline BN Pup & 13.67310 & 67.57 & 24.86 & 0.206 & 0.021 & 4.495 & 1.650 & 0.001 & 0.001 & 0.069 & 0.103 \\
\hline TT Aql & 13.75496 & 3.83 & 21.93 & 0.139 & 0.140 & 4.517 & 6.262 & 0.001 & 0.001 & 0.049 & 0.364 \\
\hline LS Pup & 14.14640 & 81.50 & 22.64 & 0.069 & 0.126 & 6.180 & 5.978 & 0.001 & 0.001 & 0.075 & 0.112 \\
\hline VW Cen & 15.03618 & -30.60 & 22.72 & 0.213 & 0.049 & 4.674 & 0.357 & 0.038 & 0.038 & 0.579 & 0.603 \\
\hline X Cyg & 16.38633 & 7.85 & 22.18 & 0.228 & 0.058 & 4.559 & 0.890 & 0.004 & 0.004 & 0.135 & 0.209 \\
\hline Y Oph & 17.12633 & -7.12 & 7.75 & 0.114 & 0.034 & 5.503 & 4.257 & 0.024 & 0.024 & 0.313 & 0.466 \\
\hline SZ Aql & 17.14071 & 6.45 & 25.38 & 0.263 & 0.039 & 4.563 & 1.735 & 0.003 & 0.003 & 0.112 & 0.167 \\
\hline CT Car & 18.05765 & 111.10 & 23.42 & 0.307 & 0.139 & 4.576 & 2.444 & 0.015 & 0.015 & 0.275 & 0.389 \\
\hline VY Car & 18.90728 & 1.86 & 22.22 & 0.272 & 0.112 & 4.518 & 2.374 & 0.029 & 0.029 & 0.417 & 0.514 \\
\hline RU Sct & 19.70062 & -5.16 & 21.14 & 0.244 & 0.014 & 4.753 & 3.027 & 0.009 & 0.009 & 0.236 & 0.330 \\
\hline RY Sco & 20.32014 & -18.00 & 15.85 & 0.050 & 0.070 & 4.077 & 4.236 & 0.045 & 0.045 & 0.643 & 0.672 \\
\hline RZ Vel & 20.39690 & 24.81 & 22.96 & 0.206 & 0.172 & 4.564 & 1.875 & 0.023 & 0.023 & 0.446 & 0.940 \\
\hline WZ Sgr & 21.84960 & -17.40 & 23.05 & 0.323 & 0.138 & 4.542 & 2.606 & 0.003 & 0.003 & 0.109 & 0.160 \\
\hline WZ Car & 23.01320 & -14.40 & 22.78 & 0.341 & 0.147 & 4.468 & 2.546 & 0.033 & 0.033 & 0.598 & 0.779 \\
\hline VZ Pup & 23.17100 & 63.52 & 23.00 & 0.303 & 0.121 & 4.446 & 2.450 & 0.001 & 0.001 & 0.068 & 0.088 \\
\hline SW Vel & 23.44313 & 23.75 & 26.88 & 0.378 & 0.200 & 4.645 & 2.962 & 0.009 & 0.009 & 0.222 & 0.304 \\
\hline X Pup & 25.96100 & 72.02 & 24.12 & 0.363 & 0.143 & 4.565 & 2.891 & 0.001 & 0.001 & 0.081 & 3.168 \\
\hline T Mon & 27.03569 & 20.67 & 22.57 & 0.345 & 0.202 & 4.580 & 2.983 & 0.005 & 0.005 & 0.152 & 0.220 \\
\hline RY Vel & 28.13117 & -10.60 & 16.18 & 0.267 & 0.127 & 4.333 & 2.464 & 0.039 & 0.039 & 0.600 & 0.602 \\
\hline KQ Sco & 28.69580 & -30.30 & 13.84 & 0.940 & 0.611 & 3.943 & 4.083 & 0.038 & 0.038 & 0.628 & 0.616 \\
\hline AQ Pup & 30.10400 & 61.02 & 23.91 & 0.276 & 0.062 & 4.733 & 3.298 & 0.001 & 0.001 & 0.066 & 0.099 \\
\hline KN Cen & 34.02964 & -37.20 & 21.71 & 0.295 & 0.341 & 3.861 & 2.323 & 0.036 & 0.036 & 0.569 & 0.655 \\
\hline$\ell \mathrm{Car}$ & 35.54804 & 2.28 & 16.77 & 0.296 & 0.150 & 4.639 & 2.949 & 0.005 & 0.005 & 0.153 & 0.225 \\
\hline U Car & 38.81233 & 0.21 & 21.00 & 0.347 & 0.116 & 4.494 & 2.531 & 0.022 & 0.022 & 0.374 & 0.503 \\
\hline RS Pup & 41.47446 & 25.91 & 19.81 & 0.444 & 0.192 & 4.615 & 3.103 & 0.008 & 0.008 & 0.185 & 0.267 \\
\hline SV Vul & 44.98561 & -1.57 & 19.54 & 0.408 & 0.197 & 4.495 & 3.108 & 0.003 & 0.003 & 0.115 & 0.167 \\
\hline GY Sge & 51.56031 & 15.96 & 13.15 & 0.301 & 0.104 & 4.766 & 3.118 & 0.014 & 0.014 & 0.250 & 0.362 \\
\hline S Vul & 68.77426 & 1.52 & 13.26 & 0.335 & 0.208 & 4.742 & 3.323 & 0.008 & 0.008 & 0.181 & 0.268 \\
\hline
\end{tabular}

and zero points for the $p$-factor relation and see where the con- $\beta_{p} \in[1.2,1.75]$ in steps of 0.05 and the slope of the relation in straints are fulfilled. We have varied the zero-point in the range the range $\alpha_{p} \in[-0.31,0.01]$ in steps of 0.03 . 
Table 5. Distances for Cepheids with HST parallax measurements from Benedict et al. (2007).

\begin{tabular}{lrrrrr}
\hline \hline$(1)$ & $\begin{array}{r}(2) \\
\log (P)\end{array}$ & $\begin{array}{r}(3) \\
d(\mathrm{HST}) \\
(\mathrm{pc})\end{array}$ & $\begin{array}{r}d(\mathrm{IRSB}) \\
(\mathrm{pc})\end{array}$ & $\begin{array}{r}(5) \\
\Delta d \\
(\mathrm{pc})\end{array}$ & $\begin{array}{r}\sigma(\Delta d) \\
(\mathrm{pc})\end{array}$ \\
\hline FF Aql & 0.650390 & 355.9 & 369.8 & 14.0 & 42.3 \\
RT Aur & 0.571489 & 416.7 & 389.0 & -27.6 & 33.0 \\
$\ell$ Car & 1.550820 & 497.5 & 517.6 & 20.1 & 47.9 \\
$\delta$ Cep & 0.729678 & 273.2 & 266.7 & -6.5 & 20.3 \\
$\beta$ Dor & 0.993131 & 318.5 & 326.6 & 8.1 & 22.0 \\
$\zeta$ Gem & 1.006500 & 359.7 & 385.5 & 25.8 & 38.3 \\
W Sgr & 0.880529 & 438.6 & 216.8 & -221.8 & 71.1 \\
X Sgr & 0.845893 & 333.3 & 322.1 & -11.2 & 28.1 \\
Y Sgr & 0.761428 & 469.5 & 436.5 & -33.0 & 75.6 \\
T Vul & 0.646934 & 526.3 & 542.0 & 15.7 & 59.4 \\
\hline
\end{tabular}

Notes. The HST distances are given in Col. 3, the IRSB distances using our preferred $p$-factor relation from Eq. (7) are given in Col. 4 and the difference in Col. 5. The uncertainty on the difference is given in the last column. $\dagger$ Not considered in the fits. This star is a known spectroscopic binary.

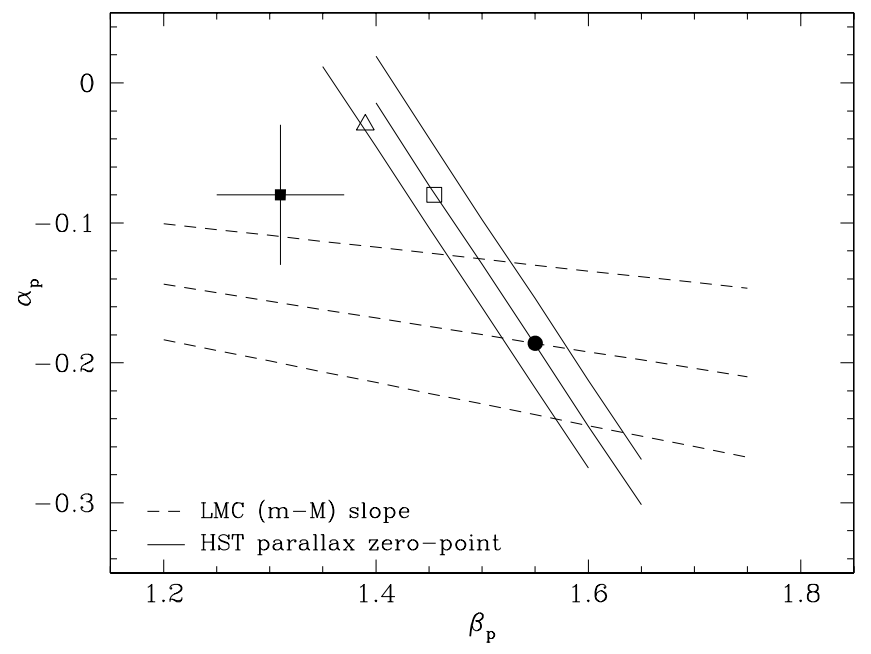

Fig. 6. Constraints on the $p$-factor relation from the HST parallax of Milky Way Cepheids (full line), and from requiring the LMC distance to be independent of the pulsation period of the stars (dashed line), see Sect. 3.4 for details. The one sigma contours are also shown with thin lines. The filled square with error bars shows the theoretical relation from Nardetto et al. (2009), the open triangle the Hindsley \& Bell (1986) relation and the filled circle the best fit. The open square shows the theoretical constraint shifted in $\beta$ to comply with the HST parallax values.

In Table 5 we have listed the stars with parallax distances from Benedict et al. $(2002,2007)$. For each pair $\left(\alpha_{p}, \beta_{p}\right)$ we have determined the IRSB distance, $d$ (IRSB), to these stars and computed the difference $\Delta(d)=d($ HST $)-d($ IRSB $)$. To weight the points independently of distance, we have normalized the values by dividing by the average distance, $d_{\mathrm{avg}}=(d(\mathrm{HST})+$ $d($ IRSB $)) / 2$., before computing the offset $\Delta(d) / d_{\text {avg }}$. We then took the mean value of these offsets and determined the values in the $\beta_{p}-\alpha_{p}$ plane where this mean offset is zero. This is a straight line which is shown in Fig. 6 as a full line with the two thin parallel lines showing the estimated $1-\sigma$ interval.

We then turn to the LMC data set and proceed as for the Milky Way sample and carry out the IRSB analysis for the same set of $\left(\alpha_{p}, \beta_{p}\right)$ values. We then look for the points where the slope of the LMC distance modulus as a function of $\log (P)$ is zero. For

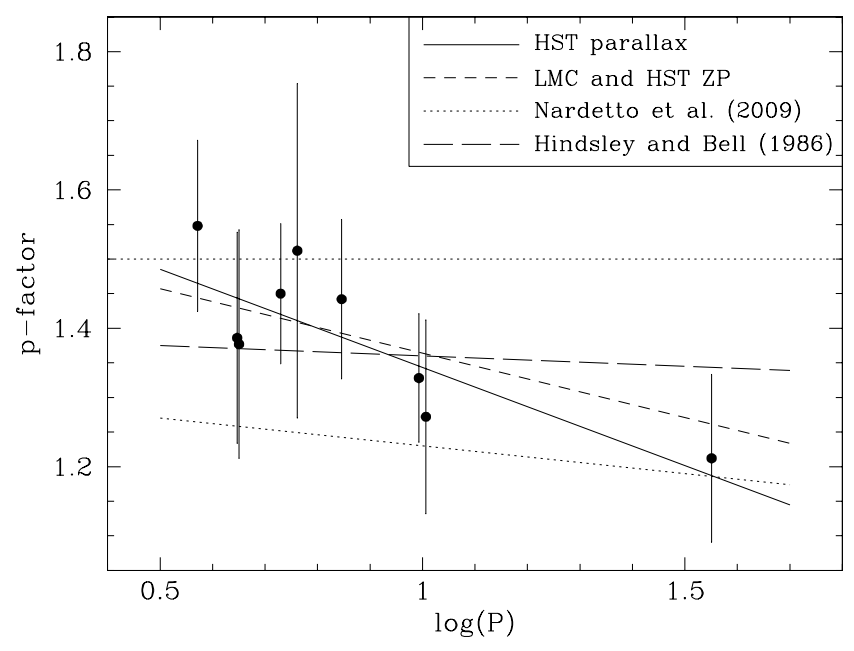

Fig. 7. The $p$-factor values derived for each of the HST parallax Cepheids with the only constraint being that the IRSB distance should agree with the parallax distance. The full line shows the linear regression fit to the points. The line labelled "LMC and HST ZP" shows our adopted relation based on the constraints from the LMC Cepheids and forcing the distance zero point to agree with the HST parallax values. The stippled line delineates the theoretical relation from Nardetto et al. (2009) and the long dashed line the classical Hindsley \& Bell (1986) relation. The horizontal line at $p=1.5$ shows the physical limit above which the theoretical $p$-factor would indicate an unphysical limbbrightening.

each individual Cepheid distance we apply the distance modulus correction $\Delta(m-M)$ from van der Marel \& Cioni (2001) to correct for the inclination of the LMC disk before determining the slope. In the $\beta_{p}, \alpha_{p}$ plane the resulting constraint is shown as a dashed line and the two thin dashed lines indicate the estimated $1-\sigma$ interval.

The best estimate is:

$$
p=1.550( \pm 0.04)-0.186( \pm 0.06) \log P .
$$

This is shown as a filled circle in Fig. 6. The $p$-factor law is even a bit steeper with period than the relation which we found earlier (Gieren et al. 2005) and it differs even more from the recent theoretical relation from Nardetto et al. (2009) which is shown as a filled square in Fig. 6. For reference the relation from Hindsley $\&$ Bell (1986) has been plotted as an open triangle in the figure. It agrees within one $\sigma$ with the HST parallax constraint but not with the constraints from the period dependence for the LMC sample. Additionally, the open square shows a relation where we have adopted the slope $\alpha_{p}=-0.08$ from Nardetto et al. (2009) but forced the zero point, $\beta_{p}$, to give agreement with the HST parallaxes.

We can look at the problem in a slightly different way and determine the $p$-factor for each of the Cepheids with measured HST parallax distances, and plot them as a function of $\log (P)$ by forcing the IRSB distance to be equal to the parallax distance. We have done this and show the results in Fig. 7. A linear fit to these values gives $p=-0.28( \pm 0.08) \log (P)+1.65( \pm 0.07)$. Within the errors this agrees with our relation (Eq. (7)). We prefer, however, to use the relation in Eq. (7) as it is based on many more stars, especially at pulsation periods longer than ten days. The linear fit is shown in the figure as well as our adopted relation (labelled LMC and HST ZP) and the Hindsley \& Bell (1986) and Nardetto et al. (2009) relations. The horizontal line at 
J. Storm et al.: Calibrating the Cepheid period-luminosity relation from the infrared surface brightness technique. I.

Table 6. Derived quantities for different adopted $p$-factor relations, $p=\beta_{p}+\alpha_{p} \log P$, for the LMC (LMC), and Milky Way (MW) samples.

\begin{tabular}{lrrrr}
\hline \hline$\alpha_{p}$ & -0.03 & -0.08 & -0.08 & -0.186 \\
$\beta_{p}$ & 1.39 & 1.31 & 1.455 & 1.550 \\
\hline $\begin{array}{l}\text { Parameter } \\
(m-M)_{0}(\mathrm{LMC})\end{array}$ & $18.50 \pm 0.04$ & $18.26 \pm 0.04$ & $18.50 \pm 0.04$ & $18.45 \pm 0.04$ \\
& & & & \\
$(m-M)_{0}(\mathrm{LMC})$ slope & $0.31 \pm 0.10$ & $0.22 \pm 0.10$ & $0.24 \pm 0.10$ & $0.00 \pm 0.10$ \\
$\Delta d(\mathrm{pc})$ & $-5 \pm 11$ & $-40 \pm 9$ & $0 \pm 10$ & $0 \pm 7$ \\
$\Delta d / d_{\mathrm{avg}}$ & $-0.02 \pm 0.02$ & $-0.11 \pm 0.02$ & $0.00 \pm 0.02$ & $0.00 \pm 0.02$ \\
& & & & \\
$a_{K}$ & $-3.58 \pm 0.09$ & $-3.49 \pm 0.08$ & $-3.50 \pm 0.09$ & $-3.33 \pm 0.09$ \\
$b_{K}$ & $-5.65 \pm 0.03$ & $-5.45 \pm 0.03$ & $-5.67 \pm 0.03$ & $-5.66 \pm 0.03$ \\
& & & & \\
$a_{V}$ & $-2.92 \pm 0.10$ & $-2.83 \pm 0.10$ & $-2.84 \pm 0.10$ & $-2.67 \pm 0.10$ \\
$b_{V}$ & $-3.95 \pm 0.03$ & $-3.73 \pm 0.03$ & $-3.97 \pm 0.03$ & $-3.96 \pm 0.03$ \\
& & & & \\
$a_{W v i}$ & $-3.51 \pm 0.11$ & $-3.42 \pm 0.12$ & $-3.43 \pm 0.11$ & $-3.26 \pm 0.12$ \\
$b_{W v i}$ & $-5.95 \pm 0.04$ & $-5.74 \pm 0.04$ & $-5.97 \pm 0.04$ & $-5.96 \pm 0.04$ \\
$a_{W j k}$ & $-3.69 \pm 0.10$ & $-3.60 \pm 0.10$ & $-3.61 \pm 0.09$ & $-3.44 \pm 0.09$ \\
$b_{W j k}$ & $-5.95 \pm 0.03$ & $-5.74 \pm 0.03$ & $-5.98 \pm 0.03$ & $-5.96 \pm 0.03$ \\
\hline
\end{tabular}

Notes. Each column corresponds to a different $p$-factor relation where the slope and zero point are given in the first two rows. The PL relations are of the form $M_{m}=a_{m} \times(\log (P)-1.0)+b_{m}$ where the index $m$ refers to the photometric band.

$p=1.5$ indicates the limit above which the $p$-factor would imply an unphysical limb-brightening instead of the expected limbdarkening. We note that for the short period stars the $p$-factor is coming close to this limit.

In Table 6 we have summarized the resulting values of true LMC modulus, the distance offset to the HST parallax data, the slope of the LMC Cepheid moduli as a function of period, for the different assumed $p$-factor relations. From this Table we can see that the Hindsley \& Bell (1986) relation leads to more than $2 \sigma$ deviation for the slope of the LMC distance modulus and thus seems to be ruled out. The Nardetto et al. (2009) relation disagrees on both the constraints, and seems to be ruled out as well. Changing the value of $\beta_{p}$ to $\beta_{p}=1.455$ brings the distance zero point into agreement with the HST parallax value, but still the distances to the LMC Cepheids are significantly dependent on the pulsation period. We thus adopt the fitted relation from Eq. (7) in the following.

In Fig. 8 we have plotted the distance difference between the HST parallax distances and our IRSB based distance when using the revised $p$-factor relation. We note that the scatter is very small and that the data are consistent with no period dependence of the differences.

\subsection{The period-luminosity relations}

Using the $p$-factor relation derived in the previous section together with the reddenings discussed in Sect. 3.2 we obtain the distances and absolute magnitudes for our Milky Way Cepheids as given in Table 7 . In that Table we present the adopted pulsation period as well as the distance modulus with the formal uncertainty from our OLS bi-sector fit (see Storm et al. 2004, for details). As discussed by Barnes et al. (2005) these uncertainties are underestimated by on average a factor of 3.4 when compared to the uncertainties returned by the Bayesian fitting technique employed in that paper. In the columns 7 to 12 we give the absolute magnitudes in the $B, V, I, J, H, \& K$ bands and in Cols. 13 and 14 we give the Wesenheit indices (Madore 1982) in the $(V-I)$ band defined as $W_{V I}=M_{V}-2.54\left(M_{V}-M_{I}\right)$ and in the near-IR $J$ and $K$ band, $W_{J K}=M_{K}-0.688\left(M_{J}-M_{K}\right)$.

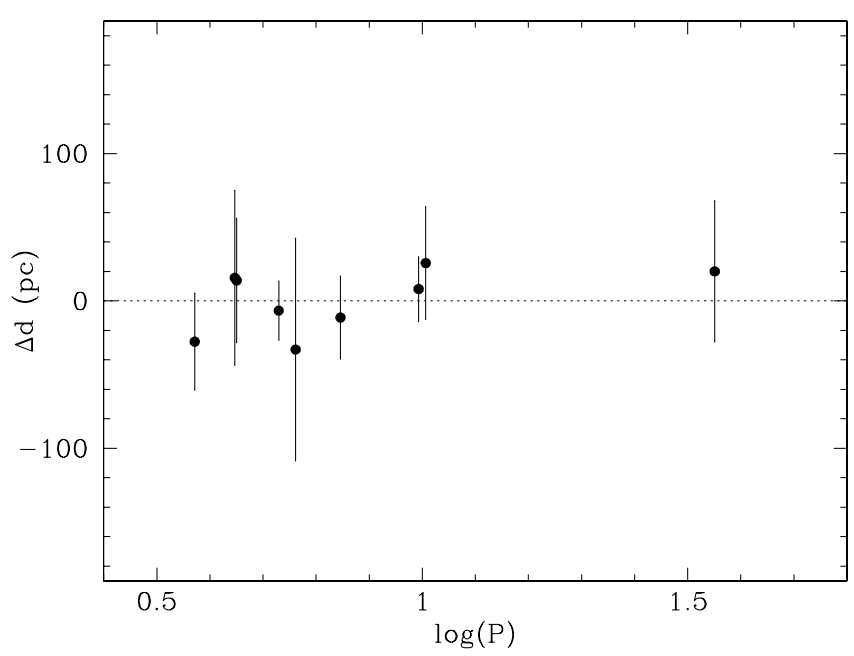

Fig. 8. The difference between the geometrical parallax distance measures for nine Cepheids from Benedict et al. (2007) and the IRSB distances values, plotted as a function of $\log P$. The revised $p$-factor relation determined in this paper has been used to calculate the IRSB distances to the stars.

In Col. 15 we give the adopted reddening value and in Col. 16 the adopted phase shift between the radial velocity data and the photometric data (see Storm et al. 2004, for details).

Using the data from Table 7 for the fundamental mode pulsators, and after eliminating the binary Cepheid W Sgr which exhibits a very poor IRSB fit, we are ready to determine the PL relations in the different bands. In Table 8 we list the resulting relations together with the observed dispersions around the fits. These are our best estimates of the Milky Way Cepheid PL relations. In Fig. 9 we plot the PL relations in the $K$, and $V$ bands as well as in the Wesenheit indices $W_{V I}$ and $W_{J K}$. We note that the dispersion around the fits range between 0.22 and 0.39 mag, the $B$-band relation showing a significantly larger dispersion than the others. For the other bands the dispersion is only weakly dependent on the wavelength suggesting that the errors on the absolute magnitudes are dominated by the distance errors rather 
A\&A 534, A94 (2011)

Table 7. Distances and intensity-averaged absolute magnitudes for the complete sample of Milky Way Cepheids based on the IRSB method as calibrated in this paper.

\begin{tabular}{|c|c|c|c|c|c|c|c|c|c|c|c|c|c|c|c|}
\hline $\begin{array}{l}\text { (1) } \\
\text { ID }\end{array}$ & $\begin{array}{r}(2) \\
\log P\end{array}$ & $\begin{array}{r}(3) \\
d \\
(\mathrm{pc})\end{array}$ & $\begin{array}{r}(4) \\
\sigma(d) \\
(\mathrm{pc})\end{array}$ & $\begin{array}{r}5) \\
(m-M)_{0} \\
(\mathrm{mag})\end{array}$ & $\begin{array}{r}(6) \\
\sigma_{(m-M)} \\
(\mathrm{mag})\end{array}$ & $\begin{array}{r}(7) \\
M_{B} \\
(\mathrm{mag})\end{array}$ & $\begin{array}{r}(8) \\
M_{V} \\
(\mathrm{mag})\end{array}$ & $\begin{array}{r}(9) \\
M_{I} \\
(\mathrm{mag})\end{array}$ & $\begin{array}{r}(10) \\
M_{J} \\
(\mathrm{mag})\end{array}$ & $\begin{array}{r}(11) \\
M_{H} \\
(\mathrm{mag})\end{array}$ & $\begin{array}{r}(12) \\
M_{K} \\
(\mathrm{mag})\end{array}$ & $\begin{array}{c}(13) \\
W_{V I} \\
(\mathrm{mag})\end{array}$ & $\begin{array}{c}(14) \\
W_{J K} \\
(\mathrm{mag})\end{array}$ & $\begin{array}{r}(15) \\
E(B-V) \\
(\mathrm{mag})\end{array}$ & $\begin{array}{r}(16) \\
\Delta \phi\end{array}$ \\
\hline SU Cas & 0.289884 & 418 & 12 & 8.10 & 0.06 & -2.53 & -2.97 & -3.52 & -3.82 & -4.05 & -4.08 & -4.37 & -4.25 & 0.259 & 0.000 \\
\hline DT Cyg & 0.397804 & 621 & 30 & 8.97 & 0.11 & -2.82 & -3.32 & -3.87 & -4.25 & -4.51 & -4.54 & -4.71 & -4.75 & 0.040 & 0.030 \\
\hline EV Sct & 0.490098 & 1766 & 82 & 11.24 & 0.10 & -2.72 & -3.21 & -3.90 & -4.19 & -4.45 & -4.46 & -4.97 & -4.64 & 0.655 & 0.045 \\
\hline SZ Tau & 0.498166 & 558 & 6 & 8.73 & 0.02 & -2.62 & -3.16 & -3.80 & -4.18 & -4.49 & -4.53 & -4.78 & -4.77 & 0.295 & -0.005 \\
\hline SS Sct & 0.564814 & 1000 & 33 & 10.00 & 0.07 & -2.21 & -2.83 & -3.55 & -3.92 & -4.24 & -4.27 & -4.65 & -4.51 & 0.325 & -0.035 \\
\hline RT Aur & 0.571489 & 389 & 4 & 7.95 & 0.02 & -2.16 & -2.69 & -3.30 & -3.75 & -4.01 & -4.05 & -4.23 & -4.26 & 0.059 & 0.005 \\
\hline QZ Nor & 0.578244 & 1346 & 38 & 10.64 & 0.06 & -2.00 & -2.62 & & -3.76 & -4.07 & -4.12 & & -4.38 & 0.263 & 0.020 \\
\hline SU Cyg & 0.584952 & 909 & 21 & 9.79 & 0.05 & -2.77 & -3.24 & -3.79 & -4.17 & -4.45 & -4.50 & -4.64 & -4.73 & 0.098 & 0.000 \\
\hline BF Oph & 0.609329 & 708 & 10 & 9.25 & 0.03 & -2.04 & -2.67 & -3.35 & -3.77 & -4.10 & -4.16 & -4.39 & -4.43 & 0.235 & 0.030 \\
\hline Y Lac & 0.635863 & 2430 & 35 & 11.93 & 0.03 & -2.97 & -3.48 & -4.08 & -4.43 & -4.74 & -4.79 & -4.99 & -5.04 & 0.217 & -0.005 \\
\hline T Vul & 0.646934 & 543 & 5 & 8.67 & 0.02 & -2.54 & -3.12 & -3.74 & -4.13 & -4.44 & -4.50 & -4.70 & -4.75 & 0.060 & 0.020 \\
\hline FF Aql & 0.650390 & 369 & 11 & 7.84 & 0.06 & -2.54 & -3.10 & -3.72 & -4.10 & -4.38 & -4.42 & -4.68 & -4.63 & 0.196 & -0.030 \\
\hline T Vel & 0.666501 & 1002 & 11 & 10.00 & 0.02 & -2.28 & -2.91 & & -4.05 & -4.40 & -4.47 & & -4.76 & 0.289 & 0.000 \\
\hline VZ Cyg & 0.687034 & 1897 & 35 & 11.39 & 0.04 & -2.68 & -3.29 & -3.98 & -4.36 & -4.68 & -4.74 & -5.04 & -5.01 & 0.266 & 0.035 \\
\hline V350 Sgr & 0.712165 & 990 & 23 & 9.98 & 0.05 & -2.86 & -3.47 & -4.15 & -4.55 & -4.89 & -4.93 & -5.20 & -5.19 & 0.299 & 0.000 \\
\hline BG Lac & 0.726883 & 1776 & 26 & 11.25 & 0.03 & -2.68 & -3.33 & -4.05 & -4.42 & -4.76 & -4.82 & -5.16 & -5.09 & 0.300 & 0.005 \\
\hline$\delta$ Cep & 0.729678 & 267 & 5 & 7.13 & 0.04 & -2.83 & -3.41 & -4.07 & -4.45 & -4.78 & -4.84 & -5.08 & -5.11 & 0.072 & 0.000 \\
\hline CV Mon & 0.730685 & 1547 & 24 & 10.95 & 0.03 & -2.40 & -2.97 & -3.75 & -4.22 & -4.58 & -4.65 & -4.94 & -4.94 & 0.722 & 0.020 \\
\hline X Lac & 0.735997 & 1852 & 38 & 11.34 & 0.04 & -3.45 & -4.02 & -4.68 & -5.05 & -5.38 & -5.42 & -5.70 & -5.68 & 0.336 & 0.040 \\
\hline V Cen & 0.739882 & 643 & 20 & 9.04 & 0.07 & -2.58 & -3.16 & -3.82 & -4.25 & -4.58 & -4.65 & -4.85 & -4.93 & 0.294 & 0.000 \\
\hline Y Sgr & 0.761428 & 437 & 15 & 8.20 & 0.07 & -2.40 & -3.07 & -3.78 & -4.25 & -4.61 & -4.67 & -4.87 & -4.95 & 0.188 & -0.030 \\
\hline CS Vel & 0.771201 & 3184 & 108 & 12.52 & 0.07 & -2.59 & -3.19 & & -4.37 & -4.71 & -4.78 & & -5.07 & 0.737 & 0.015 \\
\hline FM Aql & 0.786342 & 1182 & 28 & 10.36 & 0.05 & -3.31 & -4.00 & -4.80 & -5.12 & -5.47 & -5.52 & -6.05 & -5.79 & 0.589 & -0.010 \\
\hline XX Sgr & 0.807815 & 1042 & 42 & 10.09 & 0.09 & -2.33 & -2.92 & -3.65 & -4.11 & -4.44 & -4.47 & -4.77 & -4.73 & 0.521 & -0.025 \\
\hline BB Sgr & 0.821971 & 868 & 13 & 9.69 & 0.03 & -2.96 & -3.66 & -4.42 & -4.86 & -5.22 & -5.29 & -5.60 & -5.59 & 0.281 & 0.000 \\
\hline V Car & 0.825860 & 869 & 16 & 9.69 & 0.04 & -2.16 & -2.86 & & -4.04 & -4.40 & -4.47 & & -4.77 & 0.166 & 0.000 \\
\hline U Sgr & 0.828997 & 579 & 6 & 8.81 & 0.02 & -2.73 & -3.42 & -4.18 & -4.61 & -4.96 & -5.02 & -5.34 & -5.30 & 0.402 & 0.000 \\
\hline V496 Aql & 0.832958 & 829 & 26 & 9.59 & 0.07 & -2.37 & -3.12 & & -4.32 & -4.68 & -4.70 & & -4.96 & 0.397 & -0.055 \\
\hline X Sgr & 0.845893 & 322 & 5 & 7.54 & 0.04 & -3.23 & -3.74 & -4.35 & -4.75 & -5.06 & -5.11 & -5.28 & -5.36 & 0.237 & 0.000 \\
\hline U Aql & 0.846591 & 592 & 19 & 8.86 & 0.07 & -2.91 & -3.58 & -4.31 & -4.72 & -5.06 & -5.11 & -5.44 & -5.38 & 0.360 & 0.015 \\
\hline$\eta \mathrm{Aql}$ & 0.855930 & 255 & 4 & 7.03 & 0.09 & -2.89 & -3.55 & -4.27 & -4.70 & -5.04 & -5.10 & -5.38 & -5.38 & 0.129 & 0.000 \\
\hline W Sgr & 0.880529 & 217 & 16 & 6.68 & 0.16 & -1.73 & -2.37 & -3.03 & -3.47 & -3.80 & -3.88 & -4.05 & -4.17 & 0.108 & 0.040 \\
\hline U Vul & 0.902584 & 658 & 12 & 9.09 & 0.04 & -3.22 & -3.92 & -4.73 & -5.03 & -5.34 & -5.37 & -6.00 & -5.60 & 0.603 & 0.005 \\
\hline S Sge & 0.923352 & 671 & 16 & 9.13 & 0.05 & -3.13 & -3.84 & -4.55 & -5.00 & -5.34 & -5.41 & -5.66 & -5.70 & 0.099 & 0.000 \\
\hline GH Lup & 0.967448 & 1307 & 23 & 10.58 & 0.04 & -3.21 & -4.07 & -4.90 & -5.42 & -5.82 & -5.90 & -6.18 & -6.23 & 0.347 & -0.040 \\
\hline FN Aql & 0.976878 & 1175 & 20 & 10.35 & 0.04 & -2.81 & -3.53 & -4.36 & -4.75 & -5.12 & -5.17 & -5.63 & -5.46 & 0.486 & -0.030 \\
\hline YZ Sgr & 0.980171 & 1137 & 13 & 10.28 & 0.03 & -3.10 & -3.84 & & -5.07 & -5.44 & -5.49 & & -5.77 & 0.281 & -0.010 \\
\hline S Mus & 0.984980 & 858 & 17 & 9.67 & 0.04 & -3.30 & -3.98 & & -5.25 & -5.62 & -5.71 & & -6.02 & 0.140 & 0.010 \\
\hline S Nor & 0.989194 & 950 & 11 & 9.89 & 0.02 & -3.27 & -4.04 & -4.81 & -5.33 & -5.72 & -5.79 & -6.01 & -6.12 & 0.179 & 0.000 \\
\hline$\beta$ Dor & 0.993131 & 327 & 5 & 7.57 & 0.03 & -3.25 & -4.00 & -4.73 & -5.18 & -5.57 & -5.63 & -5.85 & -5.94 & 0.051 & 0.000 \\
\hline$\zeta \mathrm{Gem}$ & 1.006497 & 386 & 9 & 7.93 & 0.05 & -3.29 & -4.13 & & -5.33 & -5.74 & -5.81 & & -6.13 & 0.014 & 0.050 \\
\hline TW Nor & 1.032868 & 2190 & 105 & 11.70 & 0.10 & -2.96 & -3.74 & & -5.29 & -5.68 & -5.76 & & -6.08 & 1.157 & 0.015 \\
\hline Z Lac & 1.036854 & 1879 & 42 & 11.37 & 0.05 & -3.42 & -4.15 & -4.95 & -5.37 & -5.76 & -5.82 & -6.18 & -6.13 & 0.370 & 0.000 \\
\hline XX Cen & 1.039548 & 1586 & 19 & 11.00 & 0.03 & -3.35 & -4.06 & -4.80 & -5.26 & -5.63 & -5.70 & -5.94 & -6.00 & 0.271 & -0.030 \\
\hline V340 Nor & 1.052579 & 1738 & 80 & 11.20 & 0.10 & & -3.87 & & -5.23 & -5.66 & -5.74 & & -6.08 & 0.322 & -0.035 \\
\hline UU Mus & 1.065819 & 3146 & 132 & 12.49 & 0.09 & -3.26 & -4.00 & -4.79 & -5.34 & -5.74 & -5.82 & -6.02 & -6.14 & 0.404 & 0.005 \\
\hline U Nor & 1.101875 & 1289 & 39 & 10.55 & 0.07 & -3.34 & -4.08 & -4.88 & -5.43 & -5.82 & -5.89 & -6.13 & -6.21 & 0.857 & 0.000 \\
\hline SU Cru & 1.108822 & 1274 & 87 & 10.53 & 0.15 & -3.04 & -3.80 & & -5.41 & -6.05 & -6.15 & & -6.66 & 0.952 & 0.010 \\
\hline BN Pup & 1.135867 & 3817 & 82 & 12.91 & 0.05 & -3.60 & -4.37 & -5.17 & -5.67 & -6.08 & -6.15 & -6.41 & -6.48 & 0.416 & 0.020 \\
\hline TT Aql & 1.138459 & 968 & 15 & 9.93 & 0.03 & -3.33 & -4.20 & -5.11 & -5.57 & -5.98 & -6.04 & -6.51 & -6.36 & 0.435 & 0.005 \\
\hline LS Pup & 1.150646 & 4819 & 113 & 13.41 & 0.05 & -3.69 & -4.46 & -5.25 & -5.76 & -6.17 & -6.23 & -6.47 & -6.56 & 0.462 & -0.030 \\
\hline VW Cen & 1.177138 & 3417 & 68 & 12.67 & 0.04 & -2.94 & -3.84 & -4.76 & -5.43 & -5.91 & -6.01 & -6.19 & -6.42 & 0.439 & 0.000 \\
\hline X Cyg & 1.214482 & 1127 & 8 & 10.26 & 0.02 & -3.69 & -4.62 & -5.48 & -6.00 & -6.43 & -6.53 & -6.82 & -6.89 & 0.228 & 0.000 \\
\hline CD Cyg & 1.232334 & 2427 & 45 & 11.93 & 0.04 & -3.79 & -4.57 & -5.45 & -5.93 & -6.33 & -6.40 & -6.80 & -6.72 & 0.493 & 0.000 \\
\hline Y Oph & 1.233609 & 548 & 6 & 8.70 & 0.03 & -3.90 & -4.62 & -5.44 & -5.87 & -6.21 & -6.26 & -6.71 & -6.53 & 0.647 & 0.020 \\
\hline SZ Aql & 1.234029 & 2138 & 28 & 11.65 & 0.03 & -3.88 & -4.74 & -5.68 & -6.20 & -6.62 & -6.70 & -7.13 & -7.05 & 0.534 & 0.020 \\
\hline CT Car & 1.256661 & 10422 & 224 & 15.09 & 0.05 & -5.32 & -4.71 & -5.28 & -6.16 & -6.63 & -6.74 & -6.17 & -7.14 & 0.570 & 0.000 \\
\hline VY Car & 1.276818 & 1728 & 21 & 11.19 & 0.03 & -3.57 & -4.51 & -5.38 & -5.95 & -6.38 & -6.47 & -6.74 & -6.83 & 0.242 & 0.005 \\
\hline RU Sct & 1.294480 & 1895 & 40 & 11.39 & 0.04 & -4.12 & -4.85 & -5.81 & -6.47 & -6.61 & -6.66 & -7.29 & -6.79 & 0.911 & -0.010 \\
\hline RY Sco & 1.307927 & 1128 & 16 & 10.26 & 0.03 & -3.84 & -4.56 & -5.42 & -5.94 & -6.32 & -6.39 & -6.74 & -6.70 & 0.718 & 0.000 \\
\hline RZ Vel & 1.309564 & 1370 & 14 & 10.68 & 0.02 & -3.74 & -4.57 & -5.41 & -5.99 & -6.40 & -6.49 & -6.71 & -6.83 & 0.300 & 0.005 \\
\hline WZ Sgr & 1.339443 & 1774 & 35 & 11.24 & 0.04 & -3.66 & -4.62 & -5.60 & -6.25 & -6.74 & -6.84 & -7.12 & -7.25 & 0.435 & -0.015 \\
\hline WZ Car & 1.361977 & 3538 & 108 & 12.74 & 0.07 & -3.89 & -4.68 & -5.51 & -6.09 & -6.50 & -6.58 & -6.79 & -6.92 & 0.372 & 0.000 \\
\hline VZ Pup & 1.364945 & 4591 & 37 & 13.31 & 0.02 & -4.45 & -5.15 & -5.91 & -6.37 & -6.75 & -6.80 & -7.07 & -7.09 & 0.455 & -0.075 \\
\hline SW Vel & 1.370016 & 2554 & 34 & 12.04 & 0.03 & -4.21 & -5.02 & -5.87 & -6.43 & -6.85 & -6.94 & -7.18 & -7.29 & 0.344 & -0.020 \\
\hline X Pup & 1.414321 & 2720 & 24 & 12.17 & 0.02 & -4.26 & -5.03 & -5.90 & -6.39 & -6.82 & -6.88 & -7.24 & -7.22 & 0.421 & -0.105 \\
\hline T Mon & 1.431915 & 1309 & 19 & 10.59 & 0.03 & -4.04 & -5.04 & & -6.57 & -7.04 & -7.13 & & -7.52 & 0.179 & 0.000 \\
\hline RY Vel & 1.449158 & 2336 & 36 & 11.84 & 0.03 & -4.40 & -5.23 & -6.08 & -6.66 & -7.04 & -7.12 & -7.38 & -7.44 & 0.545 & 0.000 \\
\hline KQ Sco & 1.457818 & 2796 & 93 & 12.23 & 0.07 & -4.16 & -5.23 & -6.28 & -7.03 & -7.52 & -7.61 & -7.91 & -8.02 & 0.869 & -0.015 \\
\hline AQ Pup & 1.478624 & 3210 & 55 & 12.53 & 0.04 & -4.65 & -5.51 & -6.42 & -6.92 & -7.35 & -7.44 & -7.82 & -7.80 & 0.518 & -0.245 \\
\hline KN Cen & 1.531857 & 3586 & 78 & 12.77 & 0.05 & -4.65 & -5.47 & -6.33 & -7.00 & -7.48 & -7.58 & -7.66 & -7.98 & 0.791 & 0.005 \\
\hline$\ell \mathrm{Car}$ & 1.550816 & 518 & 5 & 8.57 & 0.02 & -4.18 & -5.32 & -6.30 & -6.94 & -7.45 & -7.53 & -7.83 & -7.94 & 0.146 & -0.015 \\
\hline $\mathrm{U}$ Car & 1.588970 & 1407 & 17 & 10.74 & 0.03 & -4.39 & -5.30 & -6.20 & -6.80 & -7.23 & -7.32 & -7.59 & -7.68 & 0.263 & -0.025 \\
\hline RS Pup & 1.617420 & 1810 & 30 & 11.29 & 0.04 & -4.78 & -5.76 & -6.70 & -7.28 & -7.73 & -7.82 & -8.15 & -8.19 & 0.457 & 0.035 \\
\hline SV Vul & 1.652569 & 1895 & 15 & 11.39 & 0.02 & -4.64 & -5.67 & -6.63 & -7.16 & -7.57 & -7.62 & -8.11 & -7.93 & 0.462 & 0.000 \\
\hline GY Sge & 1.708102 & 2869 & 34 & 12.29 & 0.03 & -5.39 & -6.36 & & -7.80 & -8.17 & -8.19 & & -8.46 & 1.310 & -0.025 \\
\hline S Vul & 1.837426 & 3762 & 59 & 12.88 & 0.03 & -5.36 & -6.46 & & -8.08 & -8.51 & -8.57 & & -8.91 & 0.787 & 0.050 \\
\hline
\end{tabular}

Notes. The $\sigma$ values are the nominal values returned by the bi-sector fitting algorithm. The Wesenheit indices based on the $(V, I)$ and $(J, K)$ photometry are given as well as the adopted reddening. The last column gives the adopted phase shift, $\Delta \phi$, between spectroscopic and photometric data. 
J. Storm et al.: Calibrating the Cepheid period-luminosity relation from the infrared surface brightness technique. I.
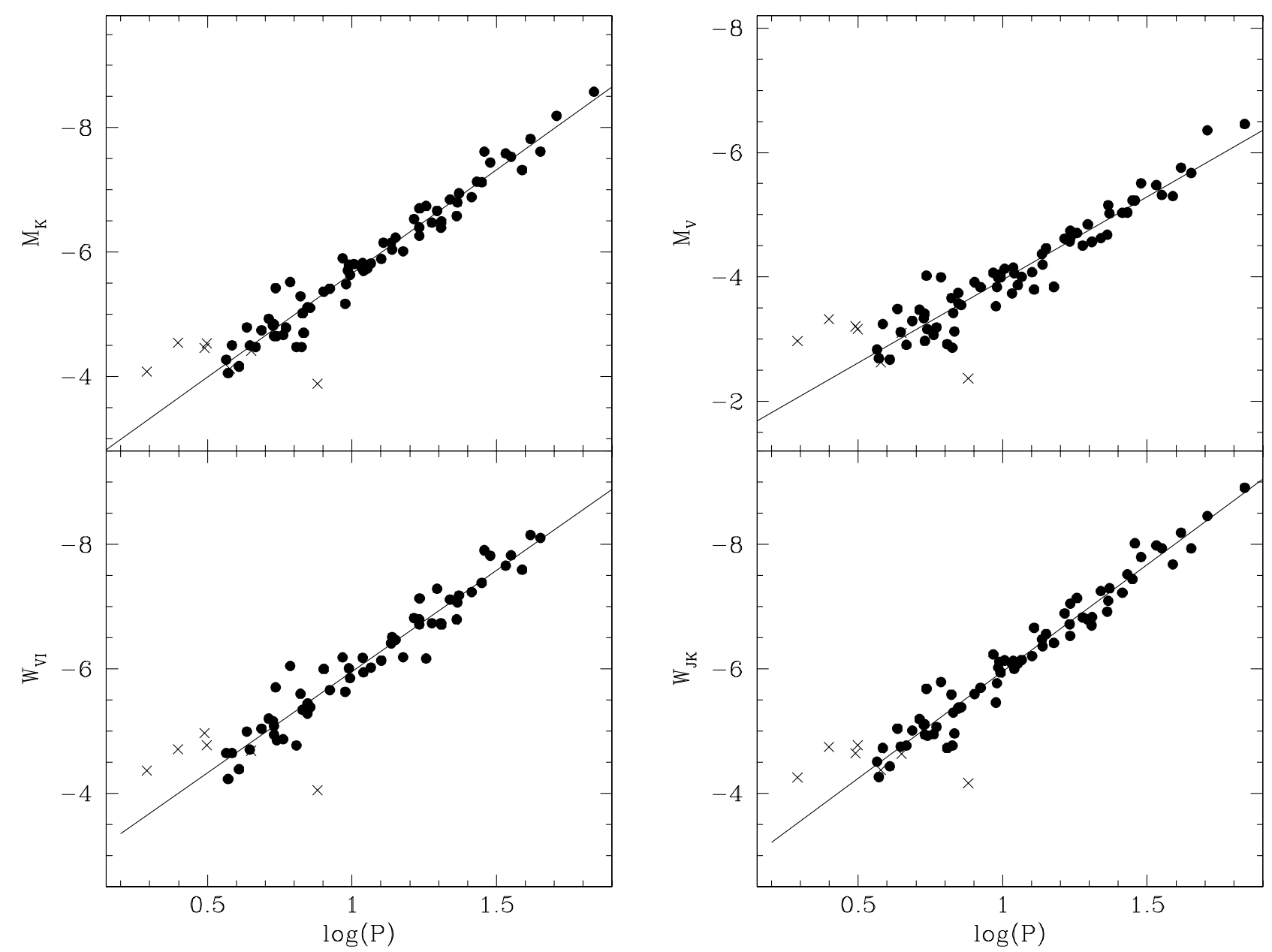

Fig. 9. The period-luminosity relations based on the IRSB luminosities for our sample of Milky Way Cepheids in the $K$ - and $V$-bands as well as in the Wesenheits indices. The filled circles represent the fundamental mode Cepheids, and the crosses indicate stars which have been disregarded in the linear regression for reasons mentioned in the text.

Table 8. Period-luminosity relations for the Milky Way Cepheids of the form $M=a \times(\log (P)-1.0)+b$.

\begin{tabular}{lccc}
\hline \hline Band & $a$ & $b$ & $\sigma$ \\
\hline$M_{B}$ & $-2.13 \pm 0.13$ & $-3.28 \pm 0.05$ & 0.39 \\
$M_{V}$ & $-2.67 \pm 0.10$ & $-3.96 \pm 0.03$ & 0.26 \\
$M_{I}$ & $-2.81 \pm 0.10$ & $-4.76 \pm 0.03$ & 0.23 \\
$W_{V I}$ & $-3.26 \pm 0.11$ & $-5.96 \pm 0.04$ & 0.26 \\
$M_{J}$ & $-3.18 \pm 0.09$ & $-5.22 \pm 0.03$ & 0.22 \\
$M_{H}$ & $-3.30 \pm 0.08$ & $-5.59 \pm 0.03$ & 0.22 \\
$M_{K}$ & $-3.33 \pm 0.09$ & $-5.66 \pm 0.03$ & 0.22 \\
$W_{J K}$ & $-3.44 \pm 0.09$ & $-5.96 \pm 0.03$ & 0.23 \\
\hline
\end{tabular}

Notes. The dispersion around the fit is also tabulated as well as the formal uncertainties on the coefficients as returned from the linear regression.

than by the intrinsic width of the PL relation and/or errors in the absorption corrections. We note that Persson et al. (2004) found a dispersion of only $0.11 \mathrm{mag}$ for the $K$-band relation in the LMC whereas in Paper II we have obtained a value of 0.22 mag, again suggesting that the dispersion in our current work is dominated by distance errors rather than intrinsic luminosity variations between Cepheids of similar periods in the sample due to the finite width of the instability strip, or errors in the reddenings.

\subsection{The combined sample}

In Paper II we show that the PL relations for the Milky Way and Large Magellanic Cloud samples are identical within the uncertainties, particularly in the near-IR bands. This means that we can combine the data from the two papers to derive a PL relation based on a total of 111 Cepheids. In Fig. 10 we have plotted the $K$-band absolute magnitudes for the Milky Way, LMC and SMC Cepheids from the two papers together. The agreement is excellent, and a linear regression to the combined sample leads to a best determination for the $K$-band PL relation of:

$M_{K}=-3.30( \pm 0.06)(\log P-1.0)-5.65( \pm 0.02)$

with a dispersion of $0.22 \mathrm{mag}$. Due to the limited metallicity dependence of this relation found in Paper II, this relation can be directly used for distance determination to galaxies with metallicities between SMC and solar.

We have listed the combined relations in the other bands, including the Wesenheit indices in Table 9, in all cases without applying any metallicity corrections to the absolute magnitudes. The relations have been used in Paper II to determine the PL relation zero-point dependence on metallicity, $\gamma$, and for convenience we have tabulated those values here as well. 
Table 9. The period-luminosity relations based on the combined samples of Milky Way, LMC and SMC Cepheids in the form $M=$ $a(\log (P)-1.0)+b$.

\begin{tabular}{lcccc}
\hline \hline Band & $\begin{array}{c}a \\
\left(\mathrm{mag} \mathrm{dex}^{-1}\right)\end{array}$ & $\begin{array}{c}b \\
(\mathrm{mag})\end{array}$ & $\begin{array}{c}\text { Std.dev. } \\
(\mathrm{mag})\end{array}$ & $\begin{array}{c}\gamma \\
\left(\mathrm{mag} \mathrm{dex}^{-1}\right)\end{array}$ \\
\hline$M_{V}$ & $-2.73 \pm 0.07$ & $-3.97 \pm 0.03$ & 0.26 & +0.09 \\
$M_{I}$ & $-2.91 \pm 0.07$ & $-4.75 \pm 0.02$ & 0.23 & -0.06 \\
$W_{V I}$ & $-3.32 \pm 0.08$ & $-5.92 \pm 0.03$ & 0.26 & -0.23 \\
$M_{J}$ & $-3.19 \pm 0.06$ & $-5.20 \pm 0.02$ & 0.22 & -0.10 \\
$M_{K}$ & $-3.30 \pm 0.06$ & $-5.65 \pm 0.02$ & 0.22 & -0.11 \\
$W_{J K}$ & $-3.38 \pm 0.06$ & $-5.96 \pm 0.02$ & 0.23 & -0.10 \\
\hline
\end{tabular}

Notes. The metallicity effect, $\gamma$, on the zero point determined in Paper II is given in the last column. The estimated uncertainty on the $\gamma$ value is estimated to be $0.10 \mathrm{mag} \mathrm{dex}^{-1}$ in Paper II.

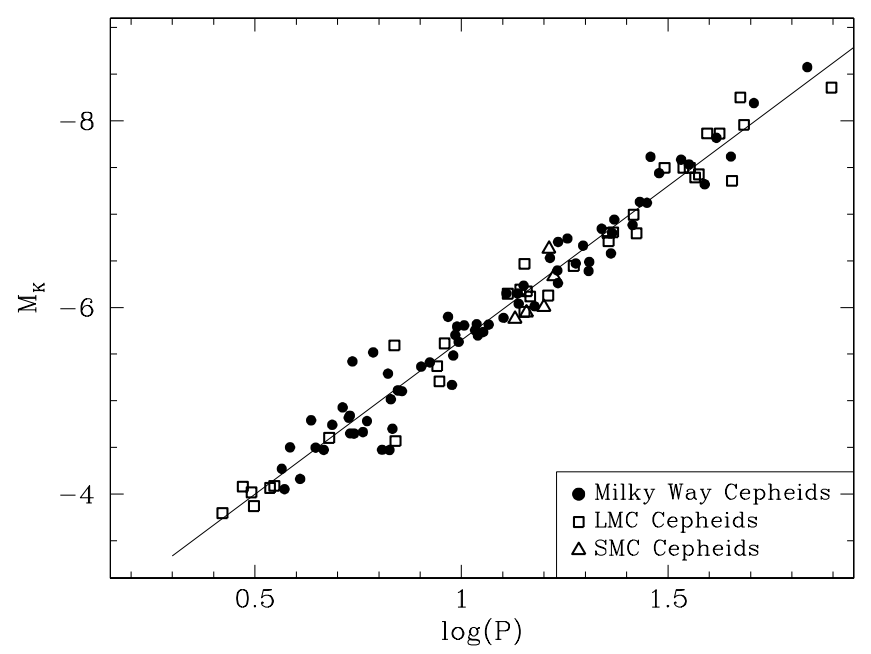

Fig. 10. The period-luminosity relation in the $K$-band for the complete sample of Milky Way, LMC and SMC Cepheids having IRSBdetermined distances in our papers. The overtone pulsators and stars which have been eliminated for other reasons as discussed earlier have been eliminated from the plot for clarity.

\section{Discussion}

The slopes of the PL relations which we find depend directly on the adopted $p$-factor relation and as we have shown the revised, empirically-determined relation is necessary to give pulsation period-independent distances to LMC Cepheids. In Paper II we further show that the adopted $p$-factor relation also reproduces the slopes of the apparent magnitude versus $\log (P)$ relations both in the near-IR and in the optical bands. The revised $p$-factor relation confirms our earlier findings (Gieren et al. 2005) based on a much smaller sample of LMC Cepheids. Still, it is at odds with the recent careful theoretical study by Nardetto et al. (2009). We do not have a ready explanation for this disagreement but suspect that it is either due to some missing physics in the theoretical approach or some previously undetected period dependence in the IRSB method, which we have eliminated through the parametrization chosen here. If the problem does not originate from the pulsational velocities, i.e. with the $p$-factor, it would have to originate with the surface-brightness calibration. The calibration is based on mostly short period Cepheids with only a single long period (35 days) Cepheid. However, Kervella et al. (2004b) show a quite convincing comparison of surfacebrightness relations for short and long period stars and the fact that the relation agrees well with the relation for static stars is also suggestive that the cause for the steep slope is not buried here. The remaining problem seems to be with the short period stars where the revised $p$-factor relation leads to values which get close to 1.5 , suggesting a uniform disk and no limb-darkening. However, the short period stars are the ones which are most numerous in the sample used by Kervella et al. (2004b) and thus the ones which have the best empirical constraints. Still we intend to make direct comparisons with interferometric studies as done by Kervella et al. (2004c) for $\ell$ Car in an attempt to understand the reason for the effect which we see. For our main purpose of investigating the effect of metallicity on the Cepheid PL relation by comparing Milky Way and Magellanic Cloud Cepheids we are working in a purely differential way so the actual $p$-factor relation cancels out as long as it is the same for both samples of stars, i.e. metal independent, which from theoretical considerations seems to be a good assumption (Nardetto et al. 2011).

Groenewegen (2007) argues for a constant $p$-factor relation based on a comparison with a subset of the HST parallax stars but the scatter in his Fig. 2 is very large. With our new high precision radial velocity data for three of the HST parallax stars, we confirm our $p$-factor relation (see Fig. 7) through excellent agreement with the full set of Benedict et al. (2007) results on a star by star basis. We also base our $p$-factor relation on a much larger sample of LMC Cepheids. This sample has a large number of stars with pulsation periods significantly longer than ten days, thus forming a much firmer basis for constraining the $p$-factor relation. In a following paper, Groenewegen (2008) discusses the use of the Nardetto et al. (2007) relation and finds no significant difference to a constant value. At the same time he finds period-luminosity relations for Milky Way Cepheids which are very similar to the relations presented in the previous section. He finds in the $K$-band a relation with a slope of $-3.38 \pm 0.08$, in good agreement with our value of $-3.33 \pm 0.09$, similarly he finds a slope in the $V$-band of $-2.60 \pm 0.09$ whereas we find a value of $-2.67 \pm 0.10$. It thus seems as if, in spite of the fact that we apply the same method, there are significant differences in the implementation of the technique which might affect the results.

Recently Molinaro et al. (2011), based on the CORS variant of the Baade-Wesselink method and using Walraven photometry for 26 galactic Cepheids and a constant $p$-factor of 1.27, found a PL relation of $M_{V}=-2.78( \pm 0.11) \log (P)-1.42( \pm 0.11)$. Again the slope is in good agreement with our relation whereas the zero point at a period of ten days differs by $0.23 \mathrm{mag}$, our value being fainter.

There are presently very few alternative routes to delineating the Milky Way PL relation apart from the Baade-Wesselink type methods. There is of course the recent direct parallaxes to ten Cepheids with the HST fine guidance sensors by Benedict et al. (2007), but this constitutes a modest sample of stars for a PL relation. The classical approach is the zero-age main sequence (ZAMS) fitting to OB associations and open clusters containing Cepheids (see e.g. Feast \& Walker 1987, and references therein). Turner (2010) rederived the Milky Way PL relation based on the ZAMS fitting to OB associations and open clusters containing Cepheids finding $M_{V}=-2.78( \pm 0.12) \log (P)-1.29( \pm 0.10)$. In Fig. 11 we compare the ZAMS fitting based distance moduli with the IRSB based moduli for the stars in common, and we find very good agreement with no significant period dependence. The unweighted mean difference is $0.12 \pm 0.06$ mag so we do find a slight zero point offset. We have excluded the star SU Cas in the comparison as it is an outlier, even if the IRSB fit appears well defined and does not indicate any obvious problem with the data. 
J. Storm et al.: Calibrating the Cepheid period-luminosity relation from the infrared surface brightness technique. I.

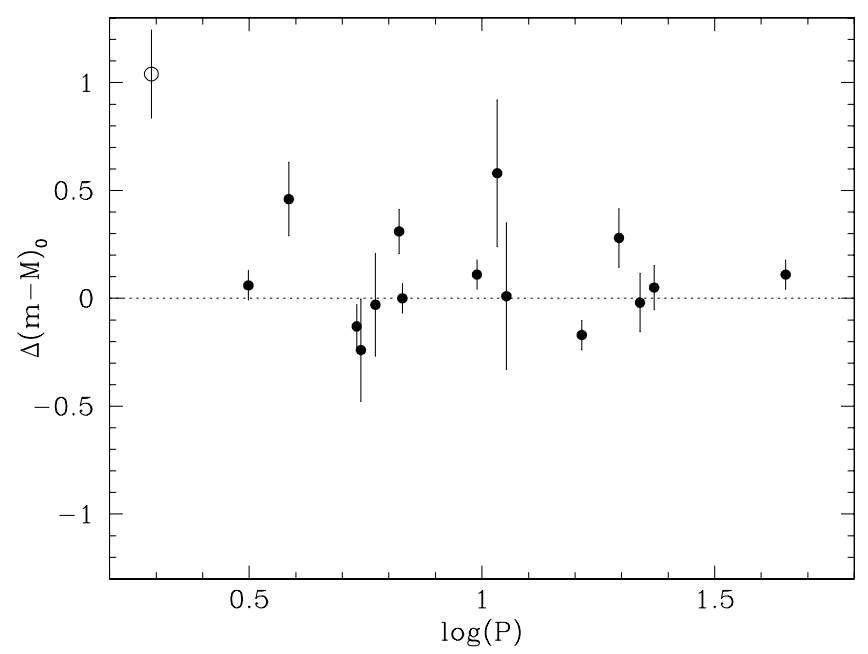

Fig. 11. The difference in derived distance modulus $\Delta(m-M)=(m-$ $M)_{\text {ZAMS }}-(m-M)_{\text {IRSB }}$. The open symbol shows the star SU Cas which has been disregarded in the comparison.

Benedict et al. (2007) found a slope of $-2.43 \pm 0.12$ in the $V$ band from the HST parallax measurements, a value which is only slightly shallower than our value of $-2.67 \pm 0.10$ and certainly not steeper than our value.

We argue that most recent investigations agree to within the errors with the slope of our relation and they disagree with the earlier findings of Sandage et al. (2004) and Storm et al. (2004), that the Milky Way PL relations are significantly steeper than the LMC relations. In fact, in Paper II we find that if anything the optical Milky Way PL relations might be slightly shallower than the LMC relations.

In Paper II we find that both the slopes and the zero-points of the near-IR PL relations are insensitive to metallicity. In addition the $K$-band PL relation is very insensitive to reddening making this relation our preferred standard candle. We argue that the best calibration of this relation is the combined $K$-band PL relation given in Table 9 with the small metallicity effect of $\gamma=-0.10 \pm 0.10 \mathrm{mag} \mathrm{dex}^{-1}$ also given in that table. We note that for most extra-galactic Cepheid samples the metallicity is close to the range from LMC to solar and the failure to correct for the metallicity effect leads to systematic errors of the order of only $0.02 \mathrm{mag}$ in the distance modulus.

In the optical bands, the LMC and Milky Way slopes are less in agreement differing by up to $0.2 \mathrm{mag} \mathrm{dex}^{-1}$ as shown in Paper II. However in the compilation by Bono et al. (2010) the slopes in the $V$ and $I$ bands for extra-galactic samples show a large spread of the order $1 \mathrm{mag} \mathrm{dex}^{-1}$, much larger than our observed difference between the LMC and Milky Way samples. The slope variations in that paper do not seem to be strongly correlated with metallicity variations so from that point of view our combined SMC, LMC, MW relation also provides the better reference relation as it is based on more stars.

\section{Conclusions and summary}

We have obtained new, accurate, radial velocity curves for fourteen Milky Way Cepheids including three Cepheids with direct parallax measures from Benedict et al. (2007), expanding the sample of Milky Way fundamental mode Cepheids to which we can determine precise IRSB distances to a total of 70 stars.

We have empirically redetermined the $p$-factor relation, which converts the observed radial velocities into pulsation velocities needed for the IRSB method, using two fundamental physical constraints. The first constraint is that the distance to LMC Cepheids should be independent of their pulsation periods, and the second constraint is that on average we should reproduce the distances to the Cepheids with parallaxes from Benedict et al. (2007). We find quite a steep relation, $p=$ $1.550( \pm 0.04)-0.186( \pm 0.06) \log (P)$ which is not easily reconciled with recent theoretical work (e.g. Nardetto et al. 2009). However, this revised relation gives rise to PL relations which are in excellent agreement with other independent determinations both for the Milky Way, as shown in the present paper, and for the LMC, as shown in Paper II.

Using the revised $p$-factor relation we have determined precise PL relations in the $V, I, J, \& K$ bands, as well as the Wesenheit indices $W_{V I}, \& W_{J K}$ for these Milky Way stars. These relations can be used for distance determination to other galaxies with solar abundance.

In Paper II we compared these relations to similar relations for a sample of LMC Cepheids and we found that the effect of metallicity on the slopes is negligible in the near-IR and small, possibly consistent with zero, in the optical bands as well. Including also a sample of SMC Cepheids we find that the zero points of the PL relations depend on metallicity to a varying degree, but in most bands the effect is small, of the order $-0.10 \pm 0.10 \mathrm{mag} \mathrm{dex}^{-1}$, which is consistent with a zero effect. Consequently we argue that it is warranted to combine the three samples of Cepheids giving us a total sample of 111 Cepheids with IRSB distances which can be used to delineate accurate absolute, universal PL relations.

Our best standard candle is the $K$-band PL relation as it is not only insensitive to reddening and shows a low intrinsic dispersion, but it also exhibits no metallicity dependence on the slope and only a weak dependence on the zero point consistent with a null effect. The $K$-band relation based on the full sample of Cepheids presented here is $M_{K}=-3.30( \pm 0.06)[\log (P)-1.0]-$ $5.65( \pm 0.02)$. The combined relation in the optical $W_{V I}$ index is $W_{V I}=-3.32( \pm 0.08)[\log (P)-1.0]-5.92( \pm 0.03)$.

Acknowledgements. This research has made use of the SIMBAD database, operated at CDS, Strasbourg, France. STELLA is funded by AIP through the State of Brandenburg and the Federal Ministry for Education and Science in Germany. It is operated jointly by AIP and the Instituto de Astrofisica de Canarias (IAC) at the Teide Observatory of the IAC. The authors thank the staff of the IAC for their great and continuous support to run STELLA. WG and GP gratefully acknowledge financial support for this work from the Chilean Center for Astrophysics FONDAP 15010003, and from the BASAL Centro de Astrofisica y Tecnologias Afines (CATA) PFB-06/2007.

\section{References}

Barnes, T. G., \& Evans, D. S. 1976, MNRAS, 174, 489

Barnes, T. G., Moffett, T. J., \& Slovak, M. H. 1988, ApJS, 66, 43

Barnes, T. G., Fernley, J. A., Frueh, M. L., et al. 1997, PASP, 109, 645

Barnes, T. G., Jeffery, E. J., Montemayor, T. J., \& Skillen, I. 2005a, ApJS, 156, 227

Barnes, T. G., Storm, J., Jefferys, W. H., Gieren, W. P., \& Fouqué, P. 2005b, ApJ, 631,572

Benedict, G. F., McArthur, B. E., Fredrick, L. W., et al. 2002, AJ, 124, 1695

Benedict, G. F., McArthur, B. E., Feast, M. W., et al. 2007, AJ, 133, 1810

Berdnikov, L. N. 1986, Variable Stars, 22, 369

Berdnikov, L. N. 1987, Variable Stars, 22, 530

Berdnikov, L. N. 1992a, A\&A Transactions, 2, 1

Berdnikov, L. N. 1992b, A\&A Transactions, 2, 31

Berdnikov, L. N. 1992c, A\&A Transactions, 2, 43

Berdnikov, L. N. 1992d, Pisma V. Astron. Zhurnal, 18, 325

Berdnikov, L. N. 1992e, Pisma V. Astron. Zhurnal, 19, 210

Berdnikov, L. N., \& Caldwell, J. A.R. 2001, J. Astr. Data, 7, 3

Berdnikov, L. N., \& Turner, D. G. 1995, Pisma V. Astron. Zhurnal, 21, 803 
Berdnikov, L. N., \& Turner, D. G. 1998, A\&A Transactions, 16, 291 Berdnikov, L. N., \& Turner, D. G. 2000, A\&A Transactions, 18, 657 Bersier, D. 2002, ApJS, 140, 465

Bersier, D., Burki, G., \& Burnet, M. 1994a, A\&AS, 108,

Bersier, D., Burki, G., Mayor, M., \& Duquennoy, A. 1994b, A\&AS, 108, 25

Bono, G., Caputo, F., Marconi, M., \& Musella, I. 2010, ApJ, 715, 277

Butler R. P., \& Bell R. A. 1997, ApJ, 480, 767

Caldwell, J. A. R., Coulson, I. M., Dean, J. F., \& Berdnikov, L. N. 2001, J. Astr. Data, 7, 4

Cardelli, J. A., Clayton, G. C., \& Mathis, J. S. 1989, ApJ, 345, 245

Carter, B. S. 1990, MNRAS, 242, 1

Coulson, I. M., \& Caldwell, J. A. R. 1985, SAAO Circulars, 9, 5

Coulson, I. M., Caldwell, J. A. R., \& Gieren, W. P. 1985, ApJS, 57, 595

Evans, N. R. 1990, PASP, 102, 551

Evans, N. R., Welch, D. L., Scarfe, C. D., \& Teays, T. J. 1990, AJ, 99, 1598

Feast, M. W., \& Walker, A. R. 1987, ARA\&A, 25, 345

Feast, M. W., Laney, C. D., Kinman, T. D., van Leeuwen, F., \& Whitelock, P. A. 2008, MNRAS, 386, 2115

Fernie, J. D., Beattie, B., Evans, N. R., \& Seager, S. 1995, IBVS No. 4148

Fouqué, P., \& Gieren, W. P. 1997, A\&A, 320, 799

Fouqué, P., Arriagada, P., Storm, J., et al. 2007, A\&A, 476, 73

Gieren, W. P. 1976, A\&A, 47, 211

Gieren, W. P. 1981a, ApJS, 46, 287

Gieren, W. P. 1981b, ApJS, 47, 315

Gieren, W. 1982, PASP, 94, 960

Gieren, W. P., Barnes, T. G., \& Moffett, T. J. 1993, ApJ, 418, 135

Gieren, W., Storm, J. Barnes, T. G., et al. 2005, ApJ, 627, 224

Gorynya, N. A., Samus', N. N., Sachkov, M. E., et al. 1998, AstL, 24, 815

Groenewegen, M. A. T. 2007, A\&A, 474, 975

Groenewegen, M. A. T. 2008, A\&A, 488, 25

Hindsley, R. B., \& Bell, R. A. 1986, PASP, 98, 881

Imbert, M. 1984, A\&AS, 58, 529

Imbert, M. 1996, A\&AS, 116, 497

Imbert, M. 1999, A\&AS, 14079

Isobe, T., Feigelson, E. D., Akritas, M. G., \& Babu, G. J. 1990, ApJ, 364, 104

Kervella, P., Nardetto, N., Bersier, D., Mourard, D., \& Coudé de Foresto, V. 2004a, A\&A, 416, 941

Kervella, P., Bersier, D., Mourard, D., et al. 2004b, A\&A, 428, 587

Kervella, P., Fouqué, P., Storm, J., et al. 2004c, ApJ, 604, L113

Kienzle, F., Moskalik, P., Bersier, D., \& Pont, F. 1999, A\&A, 341, 818

Kimeswenger, S., Lederle, C., Richichi, A., et al. 2004, A\&A, 413, 1037

Kiss, L. L. 1998a, J. Astr. Data, 4, 3

Kiss, L. L. 1998b, MNRAS, 297, 825

Laney, C. D., \& Caldwell, J. A.R. 2007, MNRAS, 377, 147

Laney, C. D., \& Stobie, R.S; 1992, A\&AS, 93, 93

Lloyd Evans, T. 1980, SAAO Circulars, 1, 257

Madore, B. F. 1975, ApJS, 29, 219

Madore, B. F. 1982, ApJ, 253, 575

Mathias, P., Gillet, D., Fokin, A. B., et al. 2006, A\&A, 457, 575
McGonegal, R., McAlary, C. W., McLaren, R. A., \& Madore, B. F. 1983, ApJ, 269,641

Mérand, A., Kervella, P., Coudé du Foresto, V., et al. 2005, A\&A, 438, L9

Metzger, M. R., Caldwell, J. A. R., McCarthy, J. K., \& Schecter, P. L. 1991 , ApJS, 76, 803

Metzger, M. R., Caldwell, J. A.R., \& Schecter, P. L. 1992, AJ, 103, 529

Moffett, T. J., \& Barnes, T. G. 1984, ApJS, 55, 389

Molinaro, R., Ripepi, V., Marconi, M., et al. 2011, MNRAS, 413, 942

Nardetto, N., Mourard, D., Kervella, P., et al. 2006, A\&A, 453, 309

Nardetto, N., Mourard, D., Mathias, P., Fokin, A., \& Gillet, D. 2007, A\&A, 471, 661

Nardetto, N., Gieren, W., Kervella, P., et al. 2009, A\&A, 502, 951

Nardetto, N., Fokin, A., Fouqué, P., et al. 2011, A\&A, in press

Nordgren, T. E., Lane, B. F., Hindsley, R. B., \& Kervella, P. 2002, AJ, 123, 3380

Ritter, A., \& Wasshüttl, A. 2004, Astron. Nachr., 325, 663

Pel, J. W. 1976, A\&AS, 24, 413

Persson, S. E., Madore, B. F., Krzemiński, W., et al. 2004, AJ, 128, 2239

Petersen, J. O. 1986, A\&A, 170, 59

Petterson, O. K. L., Cottrell, P. L., Albrow, M. D., \& Fokin, A. 2005, MNRAS, 362,1167

Pietrzyński, G., Thompson, I. B., Graczyk, D., et al. 2009, ApJ, 697, 862

Pont, F., Mayor, M., \& Burki, G. 1994, A\&A, 285, 415

Sabbey, C. N., Sasselov, D. D., Fieldus, M. S., et al. 1995, ApJ, 446, 250

Sandage, A., Tammann, G. A., \& Reindl, B. 2004, A\&A, 424, 43

Schechter, P. L., Avruch, I. M., Caldwell, J. A.R., \& Keane, M. J. 1992, AJ, 104, 1930

Shobbrook, R. R. 1992, MNRAS, 255, 486

Storm, J., Carney, B. W., Gieren, W. P., et al. 2004, A\&A, 415, 531

Storm, J., Gieren, W. P., Fouqué, P., et al. 2011, A\&A, 534, A95. (Paper II)

Strassmeier, K. G., Granzer, T., Weber, M., et al. 2004, Astron. Nachr., 325, 527

Strassmeier, K. G., Granzer, T., Weber, M., et al. 2010, Adv. Astr., 19

Sugars, B. J. A., \& Evans, N. R. 1996, AJ, 112, 1670

Szabados L. 2003, Inf. Bull. Var. Stars, No. 5394

Szewczyk, O., Pietrzyński, G., Gieren, W., et al. 2008, AJ, 136, 272

Tammann, G. A., Sandage, A., \& Reindl, B. 2003, A\&A, 404, 423

Turner, D. G. 2010, Astrophys. Space Sci., 326, 219

van der Marel, R. P., \& Cioni, M.-R. L. 2001, AJ, 122, 1807

Wallerstein, G., Jacobsen, T. S., Cottrell, P. L., Clark, M., \& Albrow, M. 1992, MNRAS, 259, 474

Walraven, J. H., Tinbergen, J., \& Walraven, T. 1964, Bull. Astr. Netherlands, 17, 520

Weber, M., Granzer, T., Strassmeier, K., \& Woche, M. 2008, Proc. SPIE, 7019, 70190L

Welch, D. L. 1985, Ph.D. Thesis, University of Toronto

Welch, D. L. 1994, AJ, 108, 1421

Welch, D. L., Wieland, F., McAlary, C. W., et al. 1984, ApJS, 54, 547

Welch, D. L., Evans, N. R., Lyons, R. W., et al. 1987, PASP, 99, 610

Wisniewski, W. Z., \& Johnson, H. L. 1968, Comm. Lunar Planetary Lab., Tucson, 7, 57 